\title{
A Stochastic SIRS Epidemic Model With Nonlinear Incidence Rate
}

\author{
Yongli Cai ${ }^{\mathrm{a}}$, Yun Kang ${ }^{\mathrm{b}}$, Weiming Wang* a \\ ${ }^{a}$ School of Mathematical Science, Huaiyin Normal University Huaian 223300, PR China \\ ${ }^{b}$ Science and Mathematics Faculty, School of Letters and Sciences, Arizona State University, Mesa, AZ \\ 85212, USA
}

\begin{abstract}
In this paper, we investigate the global dynamics of a general SIRS epidemic model with a ratio-dependent incidence rate and its corresponding stochastic differential equation version. For the deterministic model, we show that the basic reproduction number $\mathcal{R}_{0}$ determines whether there is an endemic outbreak or not: if $\mathcal{R}_{0}<1$, the disease dies out; while if $\mathcal{R}_{0}>1$, the disease persists. For the stochastic model, we show that its related reproduction number $\mathcal{R}_{0}^{S}$ can determine whether there is a unique disease-free stationary distribution or a unique endemic stationary distribution. In addition, we provide analytic results regarding the stochastic boundedness and permanence/extinction. One of the most interesting findings is that random fluctuations introduced in our stochastic model can suppress disease outbreak, which can provide us some useful control strategies to regulate disease dynamics.

Key words: Epidemic model; Basic reproduction number; Global stability; Stationary distribution; Permanence.
\end{abstract}

\section{Introduction}

According to the World Health Organization (WHO), infectious diseases are responsible for a quarter to a third of all deaths worldwide. As of 2008, four of the top ten causes of

Email addresses: caiyongli06@163.com (Yongli Cai), yun.kang@asu.edu (Yun Kang), weimingwang2003@163.com (Weiming Wang*)

* Author to whom any correspondence should be addressed. 
death were due to infectious diseases; and in low-income countries, five of the top killers were due to infectious diseases [1]. Mathematical models have been an important tool in analyzing the epidemiological characteristics of infectious diseases since the pioneer work of Kermack and McKendrick [2], which provides us useful control measures [3]. Many wellknown classic models of infectious disease population dynamics have been deterministic [4, $5,6,7]$. And the nature of epidemic growth and spread is inherently random due to the unpredictability of person-to-person contacts [8] and population is subject to a continuous spectrum of disturbances $[9,10]$. As in the early stages of an outbreak case, the infectious number will be very small, random variations alone can cause an epidemic to die out, and it is particularly important to include this randomness in models for emerging infectious diseases [8].

Stochastic models could be a more appropriate way of modeling epidemics in many circumstances $[11,12,13,14,15,16,17,18,19,20,21,22,23,24,25,26,27,28,29,30$, 31, 32, 33]. For example, stochastic models are able to take care of randomness of infectious contacts occurring in the latent and infectious periods [34]. It also has been showed that some stochastic epidemic models can provide an additional degree of realism in comparison with their deterministic counterparts [35, 36, 37, 38, 39, 40, 41, 42, 43, 44].

Many realistic stochastic epidemic models can be derived based on their deterministic formulations. Allen $[10,45]$ provided a great introduction to the methods of derivation for various types of stochastic models including stochastic differential equation (SDE) epidemic models. Ball and Neal [38] studied a general stochastic SIR model among a closed finite population, and obtained a threshold parameter that governs whether or not global epidemics can occur; Tuckwell and Williams [7] investigated the properties of a simple discrete time stochastic SIR type epidemic model, especially focusing on the influence of individuals with small population numbers and fluctuations of environment. Britton [37] gave an excellent survey on SDE epidemic models which presented the exact and asymptotic properties of a 
simple stochastic epidemic model, and was illustrated by studying effects of vaccination and inference procedures for important parameters such as the basic reproduction number and the critical vaccination coverage. Gray and coworkers [40] formulated a SDE SIS epidemic model, and proved that the model has a unique global positive solution and established conditions for extinction and persistence of infectious individuals. Yang et al. [46] investigated the ergodicity and extinction of SDE SIR and SEIR epidemic models with saturated incidence.

There are different possible approaches to including random effects in the model, both from a biological and mathematical perspective [42]. The general stochastic differential equation SIRS model introduced in this manuscript adopts the approach by Mao et al [15], which has been pursued in $[14,17,20,25,26,35,36,40,42,46]$, and assume that the parameters involved in the model always fluctuate around some average value due to continuous fluctuation in the environment. Following their approach, we will focus on a general SDE SIRS model with ratio-dependent nonlinear incidence rate where we assume that the environmental noise is proportional to the variables. The SIRS model is suitable for infectious diseases transmitted by a vector (often an invertebrate arthropod) conferring temporal acquired immunity. Since the first deterministic SIRS model with constant recruitment and disease-induced death was developed by Anderson and May [47], there are many applications of such models. For instance, the host-vector SIRS models have been proposed to study spread of Japanese Encephalitis by Mukhopadhyay and Tapaswi [48] and malaria by Tumwiine et al. [49] in human populations.

The main focus of this paper is to investigate how environment fluctuations affect disease's dynamics through studying the global dynamics of a general SIRS model with a ratiodependent transmission rate in both deterministic and stochastic versions. In particular, we aim to answer the following questions through our analytic and numerical results of SIRS models:

1. What is disease dynamics of stochastic SIRS models with ratio-dependent incidence 
rate?

2. How may different types of environmental fluctuations produce different dynamical outcomes?

The rest of this article is organized as follows: In Section 2, we derive a general SIRS deterministic model and its stochastic version with necessary definitions and preliminaries that will be used in our analysis. In Section 3, we carry out the completed global analysis of the deterministic SIRS model. In Section 4, we obtain the analytic results of dynamics of the SDE model and their related biological implications. In Section 5, we provide numerical simulations to support our answers to the research questions proposed in the above. In the last section, Section 6, we provide a brief discussion and the summary of the main results.

\section{Model Derivation}

\subsection{The deterministic SIRS model}

Let $S(t)$ be the number of susceptible individuals, $I(t)$ the number of infective individuals, and $R(t)$ the number of removed individuals at time $t$. We assume that: i) infectious disease can cause additional mortality; ii) an infectious individual can recover with a loss of immunity, and then a general SIRS model can be modeled by the following set of nonlinear differential equations (1) deterministically.

$$
\left\{\begin{array}{l}
\frac{d S}{d t}=b-d S-g(S, I)+\gamma R \\
\frac{d I}{d t}=g(S, I)-(d+\mu+\delta) I \\
\frac{d R}{d t}=\mu I-(d+\gamma) R
\end{array}\right.
$$

where $b$ is the recruitment rate of the population, $d$ the natural death rate of the population, $\mu$ the natural recovery rate of the infectious individuals, $\gamma$ the rate at which recovered individuals lose immunity and return to the susceptible class, $\delta$ the disease inducing death rate, and $g(S, I)$ the transmission of the infection or the incidence rate. 
The transmission function $g(S, I)$ plays a key role in determining disease dynamics $[50,51]$. Traditionally, the density-dependent transmission (or the bilinear incidence rate, $g(S, I)=k S I, k$ the proportionality constant) and the frequency-dependent transmission (or the standard incidence rate, $\left.g(S, I)=\frac{k S I}{S+I}\right)$ are two extreme forms of disease transmission, which have been frequently used in well-known epidemic models [52, 53]. There are several different nonlinear transmission functions proposed by researchers $[54,55,56]$. For example, Capasso and Serio [54] introduced a saturated transmission rate $g(S, I)=f(I) S$, and the infectious force $f(I)$ is a function of infectious individuals which has been used in many classic disease models. The transmission of an infectious disease may involve susceptible $S$, infectious $I$ and the total size of the population $N$, or involve $S$ and $I$ only, which is governed by the biological properties of the disease [51]. Especially, Yuan and Li [56] studied a ratio-dependent nonlinear incident rate which takes the following form (2):

$$
g(S, I)=f\left(\frac{I}{S}\right) S=\frac{k(I / S)^{l} S}{1+\alpha(I / S)^{h}}=\frac{k S^{h-l+1} I^{l}}{S^{h}+\alpha I^{h}},
$$

where $\alpha$ is the parameter which measures the psychological or inhibitory effect. It's should be noted that if $\alpha=1$ and $h=l=1$, (2) becomes the well-known frequency-dependent transmission rate $\frac{k S I}{S+I}$.

In the case of $l=1$, we can obtain the ratio-dependent transmission rate $g(S, I)$ as the following form:

$$
g(S, I)=f\left(\frac{I}{S}\right) S=\frac{k I}{1+\alpha(I / S)^{h}}=\frac{k S^{h} I}{S^{h}+\alpha I^{h}},
$$

which indicates that the transmission rate of disease is approximately governed by $k I$ when $\frac{I}{S}$ is small (e.g., at the beginning of disease's spreading) and it is approximately governed by $\frac{k}{\alpha}\left(\frac{S}{I}\right)^{h-1} S$ while $\frac{I}{S}$ is large (e.g., in the endemic when almost everyone is infected). Therefore, the ratio-dependent transmission rate (3) indeed takes accounts of the crowding effects and behavior changes during epidemics. 
In this paper, we will focus on ratio-dependent incidence rate (3). Thus, the corresponding SIRS model (1) has the following form (4)

$$
\left\{\begin{array}{l}
\frac{d S}{d t}=b-d S-\frac{k S^{h} I}{S^{h}+\alpha I^{h}}+\gamma R \\
\frac{d I}{d t}=\frac{k S^{h} I}{S^{h}+\alpha I^{h}}-(d+\mu+\delta) I \\
\frac{d R}{d t}=\mu I-(d+\gamma) R
\end{array}\right.
$$

whose state space is the first quadrant $\mathbb{R}_{+}^{3}=\{(S, I, R): S>0, I>0, R>0\}$. Denote that $\overline{\mathbb{R}}_{+}^{3}=\{(S, I, R): S \geq 0, I \geq 0, R \geq 0\}$ and a bounded set $\Gamma$

$$
\Gamma=\left\{(S, I, R) \in \mathbb{R}_{+}^{3}: \frac{b}{d+\delta} \leq S+I+R \leq \frac{b}{d}\right\} \subset \mathbb{R}_{+}^{3} .
$$

\subsection{Stochastic differential equation SIRS model}

We now turn to a continuous time SIRS model which takes random effects into account. In SIRS models, the natural death rate $d$ is one of the key parameters to disease transmission. May [57] pointed out that all the parameters involved in the population model exhibit random fluctuation as the factors controlling them are not constant. And in the real situation, the natural death rate $d$ always fluctuate around some average value due to continuous

fluctuation in the environment. In this sense, $d$ can seem as a random variable $\tilde{d}$. More precisely, in $[t, t+d t)$,

$$
-\tilde{d} d t=-d d t+\sigma\left(X_{i}\right) d W_{i}(t), i=1,2,3,
$$

here $\left(X_{1}, X_{2}, X_{3}\right)=(S, I, R)$, and $d W_{i}(t)=W_{i}(t+d t)-W_{i}(t)$ is the increment of a standard Brownian motion. For simplicity, we adopt $\sigma_{1}:=\sigma\left(X_{1}\right), \sigma_{2}:=\sigma\left(X_{2}\right), \sigma_{3}:=\sigma\left(X_{3}\right)$ are all real constants and known as the intensity of environmental fluctuations for compartments $S, I$ and $R$, respectively. The reason of adopting $\sigma_{i}(i=1,2,3)$ as the intensity of the noise for the subpopulations $S, I, R$, respectively, is considering the difference between the subpopulations mobility response to infection risks. And then, in $[t, t+d t),-\tilde{d} d t$ is normally distributed 
with mean $\mathbb{E}(-\tilde{d} d t)=-d d t$ and variance $\operatorname{Var}(-\tilde{d} d t)=\sigma_{i}^{2} d t$. Due to $\operatorname{Var}(-\tilde{d} d t)=\sigma_{i}^{2} d t \rightarrow 0$ as $d t \rightarrow 0$, this is a biologically reasonable assumption. Indeed this is a well-established way of introducing stochastic environmental noise into biologically realistic population dynamic models.

Therefore, replace $-d d t$ in equation (4) with $-\tilde{d} d t=-d d t+\sigma_{i} d W_{i}(t)(i=1,2,3)$, and for simplicity, we replace $\tilde{d}$ with $d$ again, then we can obtain the same SDE epidemic model as in (6) that is analog to its deterministic version (4) by introducing stochastic perturbation terms to the growth equations of susceptible, infectious, recovered individuals to incorporate the effect of randomly fluctuating environments:

$$
\left\{\begin{array}{l}
d S=\left(b-d S-\frac{k S^{h} I}{S^{h}+\alpha I^{h}}+\gamma R\right) d t+\sigma_{1} S d W_{1}(t), \\
d I=\left(\frac{k S^{h} I}{S^{h}+\alpha I^{h}}-(d+\mu+\delta) I\right) d t+\sigma_{2} I d W_{2}(t) \\
d R=(\mu I-(d+\gamma) R) d t+\sigma_{3} R d W_{3}(t) .
\end{array}\right.
$$

In addition, we would like to point out that Cai et al [43] studied the stochastic dynamics of the special case of model (4) when $h=1$ and $\delta=0$ and obtained the existence and uniqueness of global positive solutions, stochastic boundedness and permanence of the model. In this paper, we have broadened the scope and analysis of the dynamics of model (4) and its stochastic version.

\subsection{Preliminaries}

Let $\mathcal{B}\left(\mathbb{R}_{+}^{3}\right)$ denote the Borel $\sigma$-algebra on $\mathbb{R}_{+}^{3}$, and $\left(\mathbb{R}_{+}^{3}, \mathcal{B}\left(\mathbb{R}_{+}^{3}\right),\left\{\mathcal{F}_{t}\right\}_{t \geq 0}, \mathbb{P}\right)$ be a complete probability space with a filtration $\left\{\mathcal{F}_{t}\right\}_{t \geq 0}$ satisfying the usual conditions (i.e., it is rightly continuous and increasing while $\mathcal{F}_{0}$ contains all $\mathbb{P}$-null sets). Consider a Markov process $X(t)$ in a state space $\left(\mathbb{R}_{+}^{3}, \mathcal{B}\left(\mathbb{R}_{+}^{3}\right), \mathbb{P}\right)$ that satisfies the following Itô SDE

$$
d X(t)=u(X(t)) d t+v(X(t)) d W, X(0)=X_{0},
$$

where $W(t)$ is a standard 3-dimensional Brownian motion, $u(\cdot): \mathbb{R}_{+}^{3} \mapsto \mathbb{R}^{3}$ and $v(\cdot): \mathbb{R}_{+}^{3} \mapsto$ $\overline{\mathbb{R}}_{+}^{3 \times 3}$ are all locally Lipschitz functions. The diffusion matrix of the process $X(t)$ is defined 
as $J(X):=v(X) v^{\mathbf{T}}(X)=\left(a_{i j}(X)\right)$ (where $v^{\mathbf{T}}$ denotes the transposition of $v$ for $\mathbb{R}^{3 \times 3}$ ). Let $L$ be the second-order differential operator given by

$$
L=\sum_{i=1}^{3} u_{i}(X) \frac{\partial}{\partial X_{i}}+\frac{1}{2} \sum_{i, j=1}^{3} a_{i j}(X) \frac{\partial^{2}}{\partial X_{i} \partial X_{j}}
$$

Hence $L$ is uniformly elliptical in $\mathbb{R}_{+}^{3}$. That is, there is a positive number $M$ such that $\sum_{i, j=1}^{3} a_{i j}(X) \varphi_{i} \varphi_{j} \geq M|\varphi|^{2}$ for any $X \in \mathbb{R}_{+}^{3}$ and $\varphi \in \mathbb{R}^{3}$. The norm $|X|$, as usual, is given by $|X|=\sqrt{X_{1}^{2}+X_{2}^{2}+X_{3}^{2}}$.

It is expedient to write model (6) in the abstract form (7), and now $X(t)=(S(t), I(t), R(t))$ and

$$
u(X)=\left(\begin{array}{c}
b-d S-\frac{k S^{h} I}{S^{h}+\alpha I^{h}}+\gamma R \\
\frac{k S^{h} I}{S^{h}+\alpha I^{h}}-(d+\mu+\delta) I \\
\mu I-(d+\gamma) R
\end{array}\right), v(X)=\left(\begin{array}{ccc}
\sigma_{1} S & 0 & 0 \\
0 & \sigma_{2} I & 0 \\
0 & 0 & \sigma_{3} R
\end{array}\right), W=\left(\begin{array}{c}
W_{1} \\
W_{2} \\
W_{3}
\end{array}\right),
$$

in this sense, the diffusion matrix is $J(X)=\operatorname{diag}\left(\sigma_{1}^{2} S^{2}, \sigma_{2}^{2} I^{2}, \sigma_{3}^{2} R^{2}\right)$.

Moreover, denote $P_{t}\left(X_{0}, A\right)$ the transition probability:

$$
P_{t}\left(X_{0}, A\right)=\mathbb{P}\left(X(t) \in A \mid X(0)=X_{0}\right), \quad \forall t \in \mathbb{R}_{+} \forall X_{0} \in \mathbb{R}_{+}^{3}, \forall A \in \mathcal{B}\left(\mathbb{R}_{+}^{3}\right)
$$

To further our study, we have the following definitions:

Definition 2.1. [Stochastically ultimate boundedness [17, 23]] The solution $X(t)$ of $(7)$ is said to be stochastically ultimately bounded, if for any $\varepsilon \in(0,1)$, there is a positive constant $\varrho=\varrho(\varepsilon)$, such that for any initial value $X(0)=X_{0} \in \mathbb{R}_{+}^{3}$, the solution $X(t)$ of $(7)$ has the property that

$$
\limsup _{t \rightarrow \infty} \mathbb{P}\{|X(t)|>\varrho\}<\varepsilon
$$

Definition 2.2. [Stochastic permanence [22, 23]] The model (7) is said to be stochastically permanent if for any $\varepsilon \in(0,1)$, there exists a pair of positive constants $\varrho=\varrho(\varepsilon)$ and 
$\chi=\chi(\varepsilon)$, such that for any initial value $X_{0} \in \mathbb{R}_{+}^{3}$, the solution $X(t)$ of model $(7)$ has the property that

$$
\liminf _{t \rightarrow \infty} \mathbb{P}\{|X(t)| \leq \varrho\} \geq 1-\varepsilon, \quad \liminf _{t \rightarrow \infty} \mathbb{P}\{|X(t)| \geq \chi\} \geq 1-\varepsilon
$$

Remark 2.3. In [24], the authors gave a new definition for Stochastically Permanent: Let $x(t)=\left(x_{1}(t), \ldots, x_{n}(t)\right)^{T}$ be the solution of a stochastic system with initial value $x(0) \in$ $R_{+}^{n}$. For any $0<\varepsilon<1$, if there exists a positive constant $\delta=\delta(\varepsilon)$ such that for all $i=1, \cdots, n$,

$$
\liminf _{t \rightarrow+\infty} \mathbb{P}\left\{x_{i}(t) \geq \delta\right\} \geq 1-\varepsilon
$$

then the stochastic model (7) is said to be stochastically persistent; if there exists a positive constant $\chi=\chi(\varepsilon)$ such that for all $i=1, \cdots, n$,

$$
\liminf _{t \rightarrow+\infty} \mathbb{P}\left\{x_{i}(t) \leq \chi\right\} \geq 1-\varepsilon
$$

then the stochastic model (7) is said to be stochastically bounded from above. If the stochastic system is both stochastically persistent and stochastically bounded from above, then it is said to be stochastically permanent.

Remark 2.4. We would like to point out that, from Definition 2.2, the concept of $s$ tochastically permanent implies that the sum of all subpopulation in the system is bounded above zero and below a certain number with probability arbitrary close to 1 . And from the definition in Remark 2.3, the stochastic permanence implies that each subpopulation in the system is bounded above zero and below a certain number. And in our paper, we use Definition 2.2 for stochastic permanence.

Definition 2.5. [Stochastic extinction [40]] The infectious $I(t)$ is said to be Stochastically extinct if

$$
\mathbb{P}\left\{\lim _{t \rightarrow \infty} I(t)=0\right\}=1
$$


Definition 2.6. [Stationary distribution [58]] Denote $\mathbb{P}_{\gamma}$ the corresponding probability distribution of an initial distribution $\gamma$, which describes the initial state of (7) at $t=0$. Suppose that the distribution of $X(t)$ with initial distribution $\gamma$ converges in some sense to a distribution $\pi=\pi_{\gamma}$ (a priori $\pi$ may depend on the initial distribution $\gamma$ ), i.e.,

$$
\lim _{t \rightarrow \infty} \mathbb{P}_{\gamma}\{X(t) \in F\}=\pi(F)
$$

for all measurable $F$, then we say that (7) has a stationary distribution $\pi(\cdot)$.

We now turn our attention to the results of geometric ergodic.

Lemma 2.7. [59, 60] Assume that the following Assumptions hold:

(P1) (Minorization condition) For a compact set $U_{1} \subset \mathbb{R}_{+}^{3}$, there exist $T, \eta>0$ and a probability measure $\nu$ on $\mathbb{R}_{+}^{3}$ with $\nu\left(U_{1}\right)>0$ such that

$$
P_{T}\left(X_{0}, A\right) \geq \eta \nu(A), \forall X_{0} \in U_{1}, \forall A \in \mathcal{B}\left(\mathbb{R}_{+}^{3}\right) .
$$

(P2) (Lyapunov condition) There is a function $V: \mathbb{R}_{+}^{3} \rightarrow[1, \infty)$ with $\lim _{|X(t)| \rightarrow \infty} V(X)=\infty$ and real numbers $\beta_{1}, \beta_{2} \in(0, \infty)$ such that

$$
L V(X) \leq \beta_{1}-\beta_{2} V(X) .
$$

Then the Markov process $X(t)$ is $V$-geometrically ergodic: there exists a unique stationary distribution $\pi$ such that, for some constants $C, \lambda>0$,

$$
|\mathbb{E} g(X(t))-\pi(g)| \leq C V\left(X_{0}\right) e^{-\lambda t}, \forall X(0)=X_{0} \in \mathbb{R}_{+}^{3},
$$

for all measurable function $g \in \mathcal{G}:=\left\{\right.$ measurable $g: \mathbb{R}_{+}^{3} \rightarrow \mathbb{R}^{3}$ with $\left.|g(X)| \leq V(X)\right\}$.

About the details of the proof of Lemma 2.7, one can see Theorem 16.0.1 in [59] or Theorem 2.5 in [60].

In what follows, unless otherwise specified, the function $V(\cdot)$ represents different variables in different places.

Next, we give a classical result of the existence and uniqueness of the stationary distribution of stochastic process $X(t)$, which was established by Khasminskiui [61]. 
Lemma 2.8. [61] There exists a bounded open domain $U \subset \mathbb{R}_{+}^{3}$ with smooth boundary $\partial U$, which has the following properties:

(P3) In the domain $U$ and some neighborhood thereof, the smallest eigenvalue of the diffusion matrix $J(X)$ is bounded away from zero;

(P4) If $X_{0} \in \mathbb{R}_{+}^{3} \backslash U$, the mean time $\tau$ at which a path issuing from $x$ reaches the set $U$ is finite, and $\sup _{X_{0} \in K} \mathbb{E} \tau<\infty$ for every compact subset $K \subset \mathbb{R}_{+}^{3}$.

If assumptions above hold, then the Markov process $X(t)$ with initial value $X_{0} \in \mathbb{R}_{+}^{3}$ has a unique stationary distribution $\pi(\cdot)$. Moreover, if $f(\cdot)$ is a function integrable with respect to the measure $\pi$, then

$$
\mathbb{P}\left\{\lim _{T \rightarrow \infty} \frac{1}{T} \int_{0}^{T} f(X(t)) d t=\int_{\mathbb{R}_{+}^{3}} f(y) \pi(d y)\right\}=1 .
$$

For more details about the proof of Lemma 2.8, see, Theorems 4.1 and 4.2 in [61].

\section{Dynamics of the deterministic model}

A straightforward computation shows that model (4) is continuous and Lipschizian in $\mathbb{R}_{+}^{3}$. From the existence and uniqueness of the solution of the ordinary differential equation, there exists a unique solution of model (4) for any positive initial conditions.

The following result shows that the solutions of model (4) are bounded, and hence, lie in a compact set and are continuous for all positive time.

Lemma 3.1. $\Gamma$ defined in (5) is a positive invariant set for model (4). Moreover, every trajectory of model (4) is eventually staying in a compact subset of $\Gamma$.

The proof of Lemma 3.1 is standard (see for example, the proof of Lemma 4.1 in [18]) and hence is omitted here. In addition, from biological consideration, we can study the disease dynamics of model (4) in the bounded set $\Gamma$.

A main concern of deterministic epidemic models is to find conditions when a disease introduced into a community develops into a large outbreak, and if it does, the disease may 
become endemic. And for stochastic models, all such questions are in terms of probabilities. A useful threshold in this regard for deterministic models, called basic reproductive number, $\mathcal{R}_{0}$, is defined as the expected number of secondary infective cases per primary case in a completely susceptible subpopulation [62]. For model (4), its basic reproduction number can be easily computed as

$$
\mathcal{R}_{0}=\frac{k}{d+\mu+\delta} .
$$

And a simple calculation shows that model (4) has two equilibria points: the disease-free equilibrium $E_{0}=(b / d, 0,0)$ which exist for all parameter values, and the endemic equilibrium state $E^{*}=\left(S^{*}, I^{*}, R^{*}\right)$ with

$$
\begin{aligned}
S^{*} & =\frac{b(d+\gamma)}{d(d+\gamma)+\left(\frac{R_{0}-1}{\alpha}\right)^{1 / h}((d+\delta)(d+\gamma)+d \mu)}, \\
I^{*} & =\left(\frac{R_{0}-1}{\alpha}\right)^{1 / h} S^{*}, \\
R^{*} & =\frac{\mu}{d+\gamma}\left(\frac{R_{0}-1}{\alpha}\right)^{1 / h} S^{*},
\end{aligned}
$$

which exists provided that $\mathcal{R}_{0}>1$.

Now, we first consider the global stability of model (4) at the disease-free equilibrium $E_{0}$.

Theorem 3.2. If $\mathcal{R}_{0}<1$, the disease-free equilibrium $E_{0}$ is globally asymptotically stable, while if $\mathcal{R}_{0}>1, E_{0}$ is unstable.

Proof. If $\mathcal{R}_{0}<1$, we will use the comparison principle to show that $I(t) \rightarrow 0$ as $t \rightarrow \infty$. Due to $(S, I, R) \in \mathbb{R}_{+}^{3}$, observe from the second equation of model (4) that

$$
\frac{d I}{d t}<\left(\mathcal{R}_{0}-1\right)(d+\mu+\delta) I .
$$

The linear comparison system

$$
\frac{d \bar{I}}{d t}=\left(\mathcal{R}_{0}-1\right)(d+\mu+\delta) \bar{I}, \bar{I}(0)=I(0),
$$


which is monotone, has eigenvalues with negative real part. Consequently, $\bar{I}(t) \rightarrow 0$ as $t \rightarrow \infty$. By the comparison principle, $I(t) \rightarrow 0$ as $t \rightarrow \infty$. Thus, for any small $\varepsilon>0$, there exists $t_{0}>0$ such that for all $t \geq t_{0}, I(t) \leq \varepsilon$. From the third equation of model (4), we have

$$
\frac{d R}{d t} \leq \varepsilon \mu-(d+\gamma) R \text { for } t \geq t_{0} .
$$

Let $\bar{R}(t)$ be the solution of the following linear system

$$
\frac{d \bar{R}}{d t}=\varepsilon \mu-(d+\gamma) \bar{R} \text { for } t \geq t_{0} .
$$

With arbitrariness $\varepsilon$, we have $\bar{R}(t) \rightarrow 0$ as $t \rightarrow \infty$. Applying the comparison principle again, we have $R(t) \rightarrow 0$ as $t \rightarrow \infty$. By the same manner, we can obtain $S(t) \rightarrow b / d$ as $t \rightarrow \infty$. Hence, the disease-free equilibrium $E_{0}$ is globally asymptotically stable if $\mathcal{R}_{0}<1$.

When $\mathcal{R}_{0}>1$, the Jacobian matrix of model (4) evaluated at $E_{0}$ is

$$
J\left(E_{0}\right)=\left(\begin{array}{ccc}
-d & -k & \gamma \\
0 & k-d-\mu-\delta & 0 \\
0 & \mu & -d-\gamma
\end{array}\right),
$$

which has eigenvalues $-d<0, k-d-\mu-\delta=\left(\mathcal{R}_{0}-1\right)(d+\mu+\delta)>0,-d-\gamma<0$. Thus the disease-free equilibrium $E_{0}$ is unstable whenever $\mathcal{R}_{0}>1$.

For the stability of endemic equilibrium $E^{*}=\left(S^{*}, I^{*}, R^{*}\right)$ of model (4), we have the following theorem:

Theorem 3.3. If $\mathcal{R}_{0}>1$, the endemic equilibrium $E^{*}=\left(S^{*}, I^{*}, R^{*}\right)$ of model (4) is globally asymptotically stable.

Proof. By summing up all the equations of model (4) we find that the total population $N=S+I+R$ satisfies the following equation:

$$
\frac{d N}{d t}=b-d N-\delta I
$$


Thus, model (4) is equivalent to the following model:

$$
\left\{\begin{array}{l}
\frac{d N}{d t}=b-d N-\delta I \\
\frac{d I}{d t}=\frac{k(N-I-R)^{h} I}{(N-I-R)^{h}+\alpha I^{h}}-(d+\mu+\delta) I \\
\frac{d R}{d t}=\mu I-(d+\gamma) R
\end{array}\right.
$$

Easy to know, model (14) has a unique positive equilibrium $\left(N^{*}, I^{*}, R^{*}\right)$. And following, we will focus on that $\left(N^{*}, I^{*}, R^{*}\right)$ of model (14) is globally asymptotically stable, which will immediately prove the fact that $E^{*}=\left(S^{*}, I^{*}, R^{*}\right)$ of model (4) is globally asymptotically stable. Consider the function

$$
V(N, I, R)=\frac{1}{2}\left(N-N^{*}\right)^{2}+\varepsilon_{1}\left(I-I^{*}-I^{*} \log \frac{I}{I^{*}}\right)+\frac{1}{2} \varepsilon_{2}\left(R-R^{*}\right)^{2}
$$

where $\varepsilon_{1}$ and $\varepsilon_{2}$ are positive constants which will be determined later. Then the derivative of $V$ along the solution of model (14) is given by

$$
\begin{aligned}
\frac{d V}{d t}= & \left(N-N^{*}\right) \frac{d N}{d t}+\varepsilon_{1} \frac{I-I^{*}}{I} \frac{d I}{d t}+\epsilon_{2}\left(R-R^{*}\right) \frac{d R}{d t} \\
= & -d\left(N-N^{*}\right)^{2}-\delta\left(N-N^{*}\right)\left(I-I^{*}\right)+\varepsilon_{2} \mu\left(I-I^{*}\right)\left(R-R^{*}\right)-\varepsilon_{2}(d+\gamma)\left(R-R^{*}\right)^{2} \\
& +\varepsilon_{1} a_{1}\left(I-I^{*}\right)\left(I^{*}(N-I-R)^{h}-I^{h}\left(N^{*}-I^{*}-R^{*}\right)^{h}\right),
\end{aligned}
$$

where $a_{1}=\frac{\alpha k}{\left((N-I-R)^{h}+\alpha I^{h}\right)\left(\left(N^{*}-I^{*}-R^{*}\right)^{h}+\alpha I^{* h}\right)}>0$. An easy algebraic computation yields to

$$
\begin{aligned}
& \left(I-I^{*}\right)\left(I^{*}(N-I-R)^{h}-I^{h}\left(N^{*}-I^{*}-R^{*}\right)^{h}\right) \\
& =\left(I-I^{*}\right)\left(I^{h}\left((N-I-R)^{h}-\left(N^{*}-I^{*}-R^{*}\right)^{h}\right)-(N-I-R)^{h}\left(I^{h}-I^{* h}\right)\right) \\
& =A I^{h}\left(N-N^{*}\right)\left(I-I^{*}\right)-\left(A I^{h}+B S^{h}\right)\left(I-I^{*}\right)^{2}-A I^{h}\left(I-I^{*}\right)\left(R-R^{*}\right) .
\end{aligned}
$$

where

$$
\begin{aligned}
& A:=S^{h-1}+S^{h-2} S^{*}+S^{h-3} S^{* 2}+\cdots+S S^{* h-2}+S^{* h-1}, \\
& B:=I^{h-1}+I^{h-2} I^{*}+I^{h-3} I^{* 2}+\cdots+I I^{* h-2}+I^{* h-1} .
\end{aligned}
$$


Hence, we have

$$
\begin{aligned}
\frac{d V}{d t}= & -d\left(N-N^{*}\right)^{2}-a_{1}\left(A I^{h}+B S^{h}\right)\left(I-I^{*}\right)^{2}-\varepsilon_{2}(d+\gamma)\left(R-R^{*}\right)^{2} \\
& -\left(\delta-\varepsilon_{1} a_{1} A I^{h}\right)\left(N-N^{*}\right)\left(I-I^{*}\right)-\left(\varepsilon_{1} a_{1} A I^{h}-\varepsilon_{2} \mu\right)\left(I-I^{*}\right)\left(R-R^{*}\right) \\
= & -\left(d-\frac{\delta-\varepsilon_{1} a_{1} A I^{h}}{2}\right)\left(N-N^{*}\right)^{2}-\left(a_{1}\left(A I^{h}+B S^{h}\right)-\frac{\varepsilon_{1} a_{1} A I^{h}-\varepsilon_{2} \mu}{2}\right)\left(I-I^{*}\right)^{2} \\
& -\frac{\delta-\varepsilon_{1} a_{1} A I^{h}}{2}\left(\left(N-N^{*}\right)+\left(I-I^{*}\right)\right)^{2}-\frac{\varepsilon_{1} a_{1} A I^{h}-\varepsilon_{2} \mu}{2}\left(\left(I-I^{*}\right)+\left(R-R^{*}\right)\right)^{2} \\
& -\left(\varepsilon_{2}(d+\gamma)-\frac{\varepsilon_{1} a_{1} A I^{h}-\varepsilon_{2} \mu}{2}\right)\left(R-R^{*}\right)^{2} .
\end{aligned}
$$

Since $(S, I, R) \in \Gamma$, we get $a_{1}, A, B, I^{h}<C$, here $C$ is a positive constant. We choose $\varepsilon_{1}$ and $\varepsilon_{2}$ sufficiently small such that the coefficients of $\left(N-N^{*}\right)^{2},\left(I-I^{*}\right)^{2},\left(R-R^{*}\right)^{2},\left(\left(N-N^{*}\right)+\right.$ $\left.\left(I-I^{*}\right)\right)^{2}$ and $\left(\left(I-I^{*}\right)+\left(R-R^{*}\right)\right)^{2}$ be positive simultaneously. Thus, we have

$$
\begin{aligned}
\frac{d V}{d t} \leq & -\left(d-\frac{\delta-\varepsilon_{1} a_{1} A I^{h}}{2}\right)\left(N-N^{*}\right)^{2}-\left(a_{1}\left(A I^{h}+B S^{h}\right)-\frac{\varepsilon_{1} a_{1} A I^{h}-\varepsilon_{2} \mu}{2}\right)\left(I-I^{*}\right)^{2} \\
& -\left(\varepsilon_{2}(d+\gamma)-\frac{\varepsilon_{1} a_{1} A I^{h}-\varepsilon_{2} \mu}{2}\right)\left(R-R^{*}\right)^{2}
\end{aligned}
$$

$\leq 0$

By applying the Lyapunov-LaSalle asymptotic stability theorem [63, 64], we can obtain that the endemic equilibrium $E^{*}$ of model (4) is globally asymptotically stable.

Remark 3.4. Theorems 3.2 and 3.3 indicate that the basic reproduction number $\mathcal{R}_{0}$ is a critical parameter for model (4) determining whether there is an endemic outbreak or not, this means that the disease dies out if $\mathcal{R}_{0}<1$ and persists if $\mathcal{R}_{0}>1$. In addition, Theorem 3.3 implies that model (4) is permanent with simple dynamics whenever $\mathcal{R}_{0}>1$. However, this is not the case for the stochastic model (6) as we show in the following section.

\section{Dynamics of the SDE model}

In this section, we will focus on the dynamics of the SDE model (6). Firstly, we give some properties of the solutions to model (6), such as existence and uniqueness, geometric 
ergodicity, boundedness and permanence.

\subsection{Some properties of the solutions to (6)}

Theorem 4.1. Consider model (6), for any given initial value $X(0)=X_{0} \in \mathbb{R}_{+}^{3}$, there is a unique solution $X(t)=(S(t), I(t), R(t))$ on $t \geq 0$ and will remain in $\mathbb{R}_{+}^{3}$ with probability one.

Proof. Since the coefficients of model (6) satisfy the local Lipschitz condition, there is a unique local solution on $\left[0, \tau_{e}\right)$, where $\tau_{e}$ is the explosion time. Therefore, the unique local solution of model (6) is positive by Itô's formula. Now, let us show that this solution is global, i.e., $\tau_{e}=\infty$ a.s.

Let $n_{0}>0$ be sufficiently large for $X(t)$ lying within the interval $\left[1 / n_{0}, n_{0}\right]^{3}$. For each integer $n>n_{0}$, define the stop-times as

$$
\tau_{n}=\inf \left\{t \in\left[0, \tau_{e}\right]: \min \{S(t), I(t), R(t)\} \leq \frac{1}{n} \text { or } \max \{S(t), I(t), R(t)\} \geq n\right\} .
$$

Set $\inf \emptyset=\infty$ ( $\emptyset$ represents the empty set). $\tau_{n}$ is increasing as $n \rightarrow \infty$. Let $\tau_{\infty}=\limsup _{n \rightarrow \infty} \tau_{n}$, then $\tau_{\infty} \leq \tau_{e}$ a.s.. In the following, we need to show $\tau_{\infty}=\infty$ a.s. If this statement is violated, there exists a constant $T>0$ and an $\varepsilon \in(0,1)$ such that $\mathbb{P}\left\{\tau_{\infty} \leq T\right\}>\varepsilon$. As a consequence, there is an integer $n_{1} \geq n_{0}$ such that

$$
\mathbb{P}\left\{\tau_{n} \leq T\right\} \geq \varepsilon, n \geq n_{1} .
$$

Define a $C^{2}$-function $V: \mathbb{R}_{+}^{3} \rightarrow \mathbb{R}_{+}$by

$$
V(X)=(S-1-\ln S)+(I-1-\ln I)+(R-1-\ln R)
$$

By the Itô formula, we compute

$$
d V \triangleq L V d t+\sigma_{1}(S-1) d W_{1}(t)+\sigma_{2}(I-1) d W_{2}(t)+\sigma_{3}(R-1) d W_{3}(t)
$$


where

$$
\begin{aligned}
L V= & \left(1-\frac{1}{S}\right)\left(b-d S-\frac{k S^{h} I}{S^{h}+\alpha I^{h}}+\gamma R\right)+\left(1-\frac{1}{I}\right)\left(\frac{k S^{h} I}{S^{h}+\alpha I^{h}}-(d+\mu+\delta) I\right) \\
& +\left(1-\frac{1}{R}\right)(\mu I-(d+\gamma) R)+\frac{\sigma_{1}^{2}+\sigma_{2}^{2}+\sigma_{3}^{2}}{2} \\
= & b+3 d+\mu+\delta+\gamma+\frac{\sigma_{1}^{2}+\sigma_{2}^{2}+\sigma_{3}^{2}}{2}+\frac{k S^{h-1} I}{S^{h}+\alpha I^{h}}-\frac{k S^{h}}{S^{h}+\alpha I^{h}}-d(S+I+R) \\
& -\delta I-\frac{b}{S}-\frac{\gamma R}{S}-\frac{\mu I}{R} \\
< & b+3 d+\mu+\delta+\gamma+\frac{\sigma_{1}^{2}+\sigma_{2}^{2}+\sigma_{3}^{2}}{2}+\frac{k S^{h-1} I}{S^{h}+\alpha I^{h}} .
\end{aligned}
$$

If $S \leq I$, then $\frac{k S^{h-1} I}{S^{h}+\alpha I^{h}}=\frac{k}{\left(\frac{I}{S}\right)^{-1}+\alpha\left(\frac{I}{S}\right)^{h-1}} \leq \frac{k}{\alpha}$. While if $S>I$, then $\frac{k S^{h-1} I}{S^{h}+\alpha I^{h}}=$ $\frac{I}{S} \cdot \frac{k S^{h}}{S^{h}+\alpha I^{h}}<k$. Hence there exists a suitable constant $M>0$ independent of $S, I, R$ and $t$ such that $L V \leq M$. Substituting this inequality into (17), we see that

$$
d V(S, I, R) \leq M d t+\sigma_{1}(S-1) d B_{1}(t)+\sigma_{2}(I-1) d B_{2}(t)+\sigma_{3}(R-1) d B_{3}(t),
$$

which implies

$$
\begin{aligned}
\int_{0}^{\tau_{n} \wedge T} d V(S(r), I(r), R(r)) \leq & \int_{0}^{\tau_{n} \wedge T} M d t+\int_{0}^{\tau_{n} \wedge T} \sigma_{1}(S(r)-1) d B_{1}(r) \\
& +\int_{0}^{\tau_{n} \wedge T} \sigma_{2}(I(r)-1) d B_{2}(r)+\int_{0}^{\tau_{n} \wedge T} \sigma_{3}(R(r)-1) d B_{3}(r),
\end{aligned}
$$

where $\tau_{n} \wedge T=\min \left\{\tau_{n}, T\right\}$. Taking the expectations of the above inequality leads to

$$
\mathbb{E} V\left(S\left(\tau_{n} \wedge T\right), I\left(\tau_{n} \wedge T\right), R\left(\tau_{n} \wedge T\right)\right) \leq V(S(0), I(0), R(0))+M T
$$

Set $\Omega_{n}=\left\{\tau_{n} \leq T\right\}$ for $n \geq n_{1}$, and from (16), we have $\mathcal{P}\left(\Omega_{n}\right) \geq \varepsilon$. Note that for every $\omega \in \Omega_{n}$, there is at least one of $S\left(\tau_{n}, \omega\right) I\left(\tau_{n}, \omega\right)$ and $R\left(\tau_{n}, \omega\right)$ equaling either $n$ or $1 / n$, hence

$$
V\left(S\left(\tau_{n}, \omega\right), I\left(\tau_{n}, \omega\right), R\left(\tau_{n}, \omega\right)\right) \geq(n-1-\ln n) \wedge\left(\frac{1}{n}-1-\ln \frac{1}{n}\right) .
$$


It then follows from (18) that

$$
\begin{aligned}
V(S(0), I(0), R(0))+M T & \geq \mathbb{E}\left[I_{\Omega_{n}(\omega)} V\left(S\left(\tau_{n}\right), I\left(\tau_{n}\right), R\left(\tau_{n}\right)\right)\right] \\
& \geq \varepsilon\left((n-1-\ln n) \wedge\left(\frac{1}{n}-1-\ln \frac{1}{n}\right)\right),
\end{aligned}
$$

where $I_{\Omega_{n}}$ is the indicator function of $\Omega_{n}$. Letting $n \rightarrow \infty$, we have that

$$
\infty>V(S(0), I(0), R(0))+M T=\infty \text { a.s. }
$$

is a contradiction, then we must have $\tau_{\infty}=\infty$. Therefore, the solution of model (6) will not explode at a finite time with probability one. This completes the proof.

Remark 4.2. Theorem 4.1 shows that the solutions of model (6) will remain in $\mathbb{R}_{+}^{3}$. This is more different from that in the deterministic SIRS model (4). From Lemma 3.1, one can know that every trajectory of model (4) is eventually staying in a compact set $\Gamma \subset \mathbb{R}_{+}^{3}$.

Next, we show the existence of V-geometric ergodicity of the Markov process $X(t)=$ $(S(t), I(t), R(t))$ of model $(6)$.

Theorem 4.3. Markov process $X(t)$ of model (6) with initial value $X_{0} \in \mathbb{R}_{+}^{3}$ is $V$-geometrically ergodic.

Proof. Denote $N=S+I+R$, and define

$$
V(X(t))=N+\frac{1}{N}
$$

for $X(t) \in \mathbb{R}_{+}^{3}$. It follows that $V(X(t)) \rightarrow \infty$ as $|X(t)| \rightarrow \infty$. By Itô's formula, we have

$$
\begin{aligned}
L V(X(t)) & =b-d N-(\mu+\delta) I-\frac{b-d N-(\mu+\delta) I}{N^{2}}+\frac{\sigma_{1}^{2} S^{2}+\sigma_{2}^{2} I^{2}+\sigma_{3}^{2} R^{2}}{N^{3}} \\
& \leq-d\left(N+\frac{1}{N}\right)+b-\frac{b}{N^{2}}+\frac{2 b}{N}+\frac{(\mu+\delta) I}{N^{2}}+\frac{\sigma_{1}^{2} S^{2}+\sigma_{2}^{2} I^{2}+\sigma_{3}^{2} R^{2}}{N^{3}} \\
& \leq-d\left(N+\frac{1}{N}\right)+b-\frac{b}{N^{2}}+\frac{2 b+\mu+\delta+\sigma_{1}^{2}+\sigma_{2}^{2}+\sigma_{3}^{2}}{N} \\
& \leq C-d V(X),
\end{aligned}
$$


where

$$
C=\frac{4 b^{2}+\left(2 b+\mu+\delta+\sigma_{1}^{2}+\sigma_{2}^{2}+\sigma_{3}^{2}\right)^{2}}{4 b}
$$

Thus (P2) ( Lyapunov condition) in Lemma 2.7 holds.

Since model (6) is uniformly elliptic, Proposition 11.1 in [65] guarantees the existence of a function $p: \mathbb{R}_{+} \times \mathbb{R}_{+}^{3} \times \mathbb{R}_{+}^{3} \rightarrow(0, \infty)$ such that $p$ is jointly continuous, $p_{t}\left(X_{0}, Y\right)$ is strictly positive for all $\left(t, X_{0}, Y\right)$, and such that for all measure sets $A$

$$
P_{t}\left(X_{0}, A\right)=\int_{A} p_{t}\left(X_{0}, Y\right) d Y
$$

It follows that for any $\omega>0$, there exists a positive constant $a=a(\omega, t)>0$ so that $\inf \left\{p_{t}\left(X_{0}, Y\right): X_{0}, Y \in \mathbb{R}_{+}^{3},\left|X_{0}\right|,|Y| \leq \omega\right\} \geq a$. Assumption 1 (Minorization condition) follows immediately this, since for any measurable set $A$

$$
P_{t}(x, A)=\int_{A} p_{t}\left(X_{0}, Y\right) d Y \geq a \operatorname{Leb}\left(A \cap \mathcal{B}_{\omega}(0)\right)=a \operatorname{Leb}\left(\mathcal{B}_{\omega}(0)\right) \nu(A),
$$

where Leb is Lebesgue measure and $\nu(A)=\operatorname{Leb}\left(A \cap \mathcal{B}_{\omega}(0)\right) / \operatorname{Leb}\left(\mathcal{B}_{\omega}(0)\right)$. Thus $(\mathrm{P} 1)$ (Minirization condition) in Lemma 2.7 holds. This ends the proof.

Theorem 4.4. The solutions of model (6) are stochastically ultimately bounded and permanent for any initial value $X_{0} \in \mathbb{R}_{+}^{3}$.

Proof. Define

$$
V(t)=N(t)+\frac{1}{N(t)}
$$

where $N(t)=S(t)+I(t)+R(t)$. Applying Itô's formula and (20),

$$
\begin{aligned}
\mathbb{E}\left[e^{d t} V(t)\right] & =\mathbb{E}[V(0)]+\mathbb{E}\left[\int_{0}^{t} e^{d s}(d V(s)+L V(s)) d s\right] \\
& \leq \mathbb{E}[V(0)]+C \mathbb{E}\left[\int_{0}^{t} e^{d s} d s\right] \\
& =\mathbb{E}[V(0)]+\frac{C}{d}\left(e^{d t}-1\right) .
\end{aligned}
$$


It follows that

$$
\begin{aligned}
\mathbb{E}[V(t)] & \leq e^{-d t} \mathbb{E}[V(0)]+\frac{C}{d}\left(1-e^{-d t}\right) \\
& \leq \mathbb{E}[V(0)]+\frac{C}{d}:=H .
\end{aligned}
$$

We chose constant $\varrho$ sufficiently large such that $\frac{H}{\varrho}<1$. By Chebyshev's inequality,

$$
\mathbb{P}\left\{N+\frac{1}{N}>\varrho\right\} \leq \frac{1}{\varrho} \mathbb{E}\left[N+\frac{1}{N}\right] \leq \frac{H}{\varrho}:=\varepsilon .
$$

This implies

$$
1-\varepsilon \leq \mathbb{P}\left\{N+\frac{1}{N} \leq \varrho\right\} \leq \mathbb{P}\left\{\frac{1}{\varrho} \leq N \leq \varrho\right\} .
$$

Noting that $N^{2} \leq 3|X|^{2} \leq 3 N^{2}$, we have

$$
\mathbb{P}\left\{\frac{1}{\sqrt{3} \varrho} \leq \frac{N}{\sqrt{3}} \leq|X| \leq N \leq \varrho\right\} \geq 1-\varepsilon .
$$

According to Definition 2.1 and Definition 2.2, Model (6) are stochastically ultimately bounded and permanent. The proof is complete.

Remark 4.5. Theorem 4.4 indicates that there exists a positive constant $C$ such that

$$
A:=S^{h-1}+S^{h-2} S^{*}+S^{h-3} S^{* 2}+\cdots+S S^{* h-2}+S^{* h-1} \leq C \text { a.s. }
$$

\subsection{Stochastic disease-free dynamics}

One of the main concerns in epidemiology is how we can regulate the disease dynamics so that the disease will be eradicated in a long term. Theorem 3.2 indicates that if $\mathcal{R}_{0}<$ 1 then disease-free equilibrium $E_{0}=(b / d, 0,0)$ of the deterministic model (4) is globally asymptotically stable. However, the condition of $\mathcal{R}_{0}<1$ does not imply the extinction of disease in the stochastic model (6). In this subsection, we provide the conditions of the extinction of disease in model (6) and the related disease-free distribution. First of all, we define the basic reproduction number for the SDE model (6) as follows:

$$
\mathcal{R}_{0}^{s}:=\mathcal{R}_{0}-\frac{\sigma_{2}^{2}}{2(d+\mu+\delta)}=\mathcal{R}_{0}\left(1-\frac{\sigma_{2}^{2}}{2 k}\right) .
$$


Theorem 4.6. If $\mathcal{R}_{0}^{s}<1$, then for any given initial value $X_{0} \in \mathbb{R}_{+}^{3}, I(t)$ almost surely tends to zero exponentially.

Proof. By the Itô formula, we have

$$
d \log I(t)=\left(\frac{k S^{h}(t)}{S^{h}(t)+\alpha I^{h}(t)}-(d+\mu+\delta)-\frac{\sigma_{2}^{2}}{2}\right) d t+\sigma_{2} d W_{2}(t)
$$

Hence,

$$
\begin{aligned}
\log I(t) & =\log I_{0}+\int_{0}^{t}\left(\frac{k S(s)^{h}}{S^{h}(s)+\alpha I^{h}(s)}-(d+\mu+\delta)-\frac{\sigma_{2}^{2}}{2}\right) d s+\int_{0}^{t} \sigma_{2} d W_{2}(s) \\
& \leq \log I_{0}+\int_{0}^{t}\left(k-(d+\mu+\delta)-\frac{\sigma_{2}^{2}}{2}\right) d s+\int_{0}^{t} \sigma_{2} d W_{2}(s) \\
& =\log I_{0}+\left(k-(d+\mu+\delta)-\frac{\sigma_{2}^{2}}{2}\right) t+G(t),
\end{aligned}
$$

where $G(t)$ is a martingale defined by $G(t)=\int_{0}^{t} \sigma_{2} d W_{2}(s)$. Then $\langle G, G\rangle_{t}=\int_{0}^{t} \sigma_{2}^{2} d s=\sigma_{2}^{2} t$. By the strong law of large numbers for martingales [12], we have $\limsup _{t \rightarrow \infty} \frac{G(t)}{t}=0$ a.s. It finally follows from (25) by dividing $t$ on the both sides and then letting $t \rightarrow \infty$ that

$$
\limsup _{t \rightarrow \infty} \frac{\log I(t)}{t} \leq k-(d+\mu+\delta)-\frac{\sigma_{2}^{2}}{2}<0 \text { a.s. }
$$

which is the required assertion.

Remark 4.7. Theorem 4.6 implies that $I(t)$ goes extinct exponentially a.s. whenever $\mathcal{R}_{0}^{S}<1$ with the consequence that $R$-class also goes to extinction a.s.. In summary, Theorem 4.6 gives a condition when solutions of model (6) are converging to the disease-free dynamics a.s.

Next, we will focus on the existence of disease-free distribution of stochastic model (6) when $\mathcal{R}_{0}^{S}<1$. First of all, we give the following lemmas.

Lemma 4.8. Consider the equation

$$
\left\{\begin{array}{l}
d u(t)=(b-d u(t)) d t+\sigma_{1} u(t) d W_{1}(t) \\
u(0)=u_{0}
\end{array}\right.
$$


then the distribution of the process $\psi:=\log u(t)$ converges in distribution to a stationary distribution which has the density

$$
f_{*}(\psi)=K_{1} \exp \left\{-\frac{1}{\sigma_{1}^{2}}\left(2 b \exp \{-\psi\}+2 d \psi+\sigma_{1}^{2} \psi\right)\right\},
$$

where $K_{1}=\left(\int_{0}^{\infty} \exp \left\{-\frac{1}{\sigma_{1}^{2}}\left(2 b \exp \{-\psi\}+2 d \psi+\sigma_{1} \psi\right)\right\} d \psi\right)^{-1}$.

Proof. By putting $u(t)=\exp \{\psi(t)\}$, we have

$$
d \psi(t)=\left(b \exp \{-\psi(t)\}-d-\frac{\sigma_{1}^{2}}{2}\right) d t+\sigma_{1} d W_{1}(t) .
$$

Then the equation above has a unique distribution which has a density $f_{*}(\psi)$ satisfying the Fokker-Planck equation

$$
\frac{1}{2} \sigma_{1}^{2} \frac{d^{2} f_{*}(\psi)}{d \psi^{2}}-\frac{d}{d \psi}\left(\left(b \exp \{-\psi\}-d-\frac{\sigma_{1}^{2}}{2}\right) f_{*}(\psi)\right)=0 .
$$

The general solution of equation (29) is

$$
\begin{aligned}
f_{*}(\psi)= & \exp \left\{-\frac{1}{\sigma_{1}^{2}}\left(2 b \exp \{\psi\}+2 d \psi+\sigma_{1}^{2} \psi\right)\right\} \\
& \left(K_{1}-K_{2} \int_{0}^{r} \exp \left\{\frac{1}{\sigma_{1}^{2}}\left(2 b \exp \{\psi\}+2 d \psi+\sigma_{1}^{2} \psi\right)\right\} d r\right),
\end{aligned}
$$

where $K_{1}, K_{2}$ are two constants. It follows from the conditions

$$
f_{*}(\psi) \geq 0, \quad \int_{0}^{\infty} f_{*}(\psi) d \psi=1,
$$

that $K_{2}=0$ and

$$
K_{1}=\left(\int_{0}^{\infty} \exp \left\{-\frac{1}{\sigma_{1}^{2}}\left(2 b \exp \{-\psi\}+2 d \psi+\sigma_{1}^{2} \psi\right)\right\} d \psi\right)^{-1} .
$$

Therefore, by the existence of a distribution [66], $\psi(t)=\log u(t)$ converges to the measure with density $f_{*}(\psi)$ as $t \rightarrow \infty$. 
Lemma 4.9. Consider model (27), the distribution of the process $u(t)$ converges to a stationary distribution which has a density

$$
p(u)=\frac{\left(\frac{2 b}{\sigma_{1}^{2}}\right)^{\frac{2 d}{\sigma_{1}^{2}}+1}}{\Gamma\left(\frac{2 d}{\sigma_{1}^{2}}+1\right)} u^{-\frac{2 d}{\sigma_{1}^{2}}-2} e^{-\frac{2 b}{\sigma_{1}^{2} u}}
$$

where $\Gamma(x)=\int_{0}^{\infty} t^{x-1} e^{-t} d t$. Moreover, if $\frac{2 b d}{b+d}<\sigma_{1}^{2}<2 d$, the mean and variance of the stationary distribution of the process $u(t)$ are, respectively,

$$
\mathbb{E}[u]=\frac{b}{d}, \quad \operatorname{Var}[u]=\frac{b}{d}\left(\frac{\sigma_{1}^{2}}{2 d-\sigma_{1}^{2}}-\frac{b}{d}\right) .
$$

Proof. The stationary Fokker-Plank equation of (27) is

$$
\frac{d}{d u}((b-d u) p(u))-\frac{1}{2} \frac{d^{2}}{d u^{2}}\left(\sigma^{2} u^{2} p(u)\right)=0
$$

with $p(u)$ a $C^{2}$-function. Rewrite Eq. (32) in the following simpler form

$$
\frac{d y}{d u}-a(u) y=-c
$$

where $y=\sigma_{1}^{2} u^{2} p(u), a(u)=\frac{2(b-d u)}{\sigma_{1}^{2} u^{2}}$ and $c$ is a constant. The solution of (33) is

$$
y(u)=A(u)\left(K_{1}-c \int_{1}^{u} \frac{1}{A(z)} d z\right)
$$

with $A(z)=e^{\int_{1}^{z} a(\tau) d \tau}$ and $K_{1}$ constant. It follows that

$$
p(u)=\frac{A(u)}{\sigma^{2} u^{2}}\left(k-c \int_{1}^{u} \frac{1}{A(z)} d z\right) .
$$

Observing that

$$
\int_{1}^{u} a(\tau) d \tau=-\frac{2 b}{\sigma_{1}^{2} u}-\frac{2 d \ln u}{\sigma_{1}^{2}}+c_{1}
$$

with $c_{1}$ constant and therefore $A(u)=c_{2} u^{-\frac{2 d}{\sigma_{1}^{2}}} e^{-\frac{2 b}{\sigma_{1}^{2} u}}$. It follows from the conditions $p(u) \geq$ $0, \quad \int_{0}^{\infty} p(u) d u=1$, that $c=0$ and $K_{1}=\left(\int_{0}^{\infty} \frac{c_{2}}{\sigma_{1}^{2}} u^{-\frac{2 d}{\sigma_{1}^{2}}-2} e^{-\frac{2 b}{\sigma_{1}^{2} u}}\right)^{-1}$. We can conclude that $p(u)$ has the form (28). Moreover,

$$
\mathbb{E}[u]=\int_{0}^{\infty} u p(u)=\frac{2 b}{\sigma^{2}} \frac{\Gamma\left(\frac{2 d}{\sigma^{2}}\right)}{\Gamma\left(\frac{2 d}{\sigma^{2}}+1\right)}=\frac{b}{d}
$$


and

$$
\mathbb{E}\left[u^{2}\right]=\int_{0}^{\infty} u^{2} p(u)=\frac{2 b}{\sigma^{2}} \frac{\Gamma\left(\frac{2 d}{\sigma^{2}}-1\right)}{\Gamma\left(\frac{2 d}{\sigma^{2}}+1\right)}=\frac{b \sigma^{2}}{d\left(2 d-\sigma^{2}\right)},
$$

hence,

$$
\operatorname{Var}[u]=\mathbb{E}\left[u^{2}\right]-\mathbb{E}^{2}[u]=\frac{b}{d}\left(\frac{\sigma^{2}}{2 d-\sigma^{2}}-\frac{b}{d}\right)
$$

Lemma 4.10. If $\mathcal{R}_{0}^{s}<1$, then the distribution of the process $\log X(t)$ in (6) converges to the stationary distribution which has the density defined as in (28).

Proof. Putting $S(t)=\exp \{\xi(t)\}, I(t)=\exp \{\eta(t)\}, R(t)=\exp \{\zeta(t)\}$, and substituting these transformations into model (6) we obtain

$$
\left\{\begin{array}{l}
d \xi(t)=f_{1}(\xi, \eta, \zeta) d t+\sigma_{1} d W_{1}(t), \\
d \eta(t)=f_{2}(\xi, \eta, \zeta) d t+\sigma_{2} d W_{2}(t), \\
d \zeta(t)=f_{3}(\xi, \eta, \zeta) d t+\sigma_{3} d W_{3}(t),
\end{array}\right.
$$

where

$$
\begin{aligned}
& f_{1}(\xi, \eta, \zeta)=b \exp \{-\xi(t)\}-\frac{\sigma_{1}^{2}}{2}-d-\frac{k \exp \{(h-1) \xi(t)+\eta(t)\}}{\exp \{h \xi(t)\}+\alpha \exp \{h \eta(t)\}}+\gamma \exp \{\zeta(t)-\xi(t)\}, \\
& f_{2}(\xi, \eta, \zeta)=\frac{k \exp \{h \xi(t)\}}{\exp \{h \xi(t)\}+\alpha \exp \{h \eta(t)\}}-(d+\mu+\delta)-\frac{\sigma_{2}^{2}}{2}, \\
& f_{3}(\xi, \eta, \zeta)=\mu \exp \{\eta(t)-\zeta(t)\}-(d+\gamma)-\frac{\sigma_{3}^{2}}{2}
\end{aligned}
$$

According to Theorem 4.6, if $\mathcal{R}_{0}^{s}<1$, for every $\omega \in \Omega$, we get $\lim _{t \rightarrow \infty} \eta(t)=-\infty$, then there exists $t_{0} \geq 0$ such that for all $t \geq t_{0}, \mu \exp \{\eta(t)\} \leq \frac{1}{2}(d+\gamma)$. Now, from the third equation of model (36), we have

$$
\begin{aligned}
d \zeta(t) & =\left(\mu \exp \{\eta(t)-\zeta(t)\}-(d+\gamma)-\frac{\sigma_{3}^{2}}{2}\right) d t+\sigma_{3} d W_{3}(t) \\
& \leq\left(\mu \exp \{\eta(t)\}-(d+\gamma)-\frac{\sigma_{3}^{2}}{2}\right) d t+\sigma_{3} d W_{3}(t),
\end{aligned}
$$


and

$$
\begin{aligned}
\zeta(t) & \leq \zeta(0)-\left(d+\gamma+\frac{\sigma_{3}^{2}}{2}\right) t+\mu \int_{0}^{t} \exp \{\eta(s)\} d s+\sigma_{3} W_{3}(t) \\
& \leq \zeta(0)-\frac{1}{2}\left(d+\gamma+\sigma_{3}^{2}\right) t+\mu \int_{0}^{t_{0}} \exp \{\eta(s)\} d s+\sigma_{3} W_{3}(t) \text { a.s.. }
\end{aligned}
$$

This implies that $\lim _{t \rightarrow \infty} \zeta(t)=-\infty$ for every $\omega \in \Omega$. To end the proof of the theorem, we still need to prove that $\xi(t)$ converges in distribution to a stationary solution $\bar{\xi}$ of the following equation

$$
\left\{\begin{array}{l}
d \bar{\xi}(t)=\left(b \exp \{-\bar{\xi}(t)\}-d-\frac{\sigma_{1}^{2}}{2}\right) d t+\sigma_{1} d W_{1}(t), \\
\bar{\xi}(0)=\xi_{0} .
\end{array}\right.
$$

By Lemma 4.8, the distribution of $\bar{\xi}$ has the density $f_{*}(x)$ defined by (28) and

$$
\lim _{t \rightarrow \infty} \bar{\xi}(t)=\bar{\xi}, \text { in distribution. }
$$

We can prove that

$$
\lim _{t \rightarrow \infty}(\bar{\xi}(t)-\xi(t))=0, \text { in probability. }
$$

Give arbitrary small $\varepsilon$. Consider the following equation

$$
\left\{\begin{array}{l}
d \bar{\xi}_{\varepsilon}(t)=\left((b+\varepsilon) \exp \left\{-\bar{\xi}_{\varepsilon}(t)\right\}-d-\frac{\sigma_{1}^{2}}{2}\right) d t+\sigma_{1} d W_{1}(t), \\
\bar{\xi}_{\varepsilon}(0)=\xi_{0} .
\end{array}\right.
$$

According to the comparison theorem, we have

$$
\bar{\xi}(t) \leq \bar{\xi}_{\varepsilon}(t) \wedge \xi(t), \text { a.s. }
$$

It follows from $\lim _{t \rightarrow \infty} \zeta(t)=-\infty$ a.s that for arbitrary small $\bar{\varepsilon} \in(0, \varepsilon)$, there exist $t_{0}$ and a set $\Omega_{\bar{\varepsilon}}$ such that $\mathbb{P}\left(\Omega_{\bar{\varepsilon}}\right)>1-\bar{\varepsilon}$ and $\gamma \exp \{\zeta(t)\}<\bar{\varepsilon}$ for $t \geq t_{0}$ and $\omega \in \Omega_{\bar{\varepsilon}}$. Thus $\xi(t) \leq \bar{\xi}_{\varepsilon}(t)$ on $\Omega_{\bar{\varepsilon}}$ for all $t \geq t_{0}$. This implies $\lim \inf _{t \rightarrow \infty}\left(\bar{\xi}_{\varepsilon}(t)-\xi(t)\right) \geq 0$ on $\omega \in \Omega_{\bar{\varepsilon}}$. Letting $\bar{\varepsilon} \rightarrow 0$ yields

$$
\liminf _{t \rightarrow \infty}\left(\bar{\xi}_{\varepsilon}(t)-\xi(t)\right)=0, \text { a.s.. }
$$


Now, equations (37) and (40) can be solved explicitly and give

$$
\begin{aligned}
& \exp \left\{\bar{\xi}_{\varepsilon}(t)\right\}=C(t)\left(\exp \left\{\xi_{0}\right\}+(b+\varepsilon) \int_{0}^{t} \exp \left\{\left(d+\frac{\sigma_{1}^{2}}{2}\right) s-\sigma_{1} B_{1}(s)\right\} d s\right), \\
& \exp \{\bar{\xi}(t)\}=C(t)\left(\exp \left\{\xi_{0}\right\}+b \int_{0}^{t} \exp \left\{\left(d+\frac{\sigma_{1}^{2}}{2}\right) s-\sigma_{1} B_{1}(s)\right\} d s\right),
\end{aligned}
$$

where $C(t)=\exp \left\{-\left(d+\frac{\sigma_{1}^{2}}{2}\right) t+\sigma_{1} B_{1}(t)\right\}$. Since $B_{1}(s) \sim N(0, s)$, we have $\mathbb{E} \exp \left\{\sigma_{1} B_{1}(s)\right\}=$ $\exp \left\{\frac{1}{2} \sigma_{1}^{2} s\right\}$, then

$$
\begin{aligned}
\mathbb{E}\left|\exp \left\{\bar{\xi}_{\varepsilon}(t)\right\}-\exp \{\bar{\xi}(t)\}\right| & =\varepsilon \int_{0}^{t} \mathbb{E}\left(\exp \left\{-\left(d+\frac{\sigma_{1}^{2}}{2}\right)(t-s)+\sigma_{1}\left(B_{1}(t)-B_{1}(s)\right)\right\}\right) d s \\
& =\frac{\varepsilon}{d}(1-\exp \{-d t\}) .
\end{aligned}
$$

From the equality above, it is easy to see that

$$
\lim _{t \rightarrow \infty} \mathbb{E}\left|\exp \left\{\bar{\xi}_{\varepsilon}(t)\right\}-\exp \{\bar{\xi}(t)\}\right|=\frac{\varepsilon}{d} .
$$

Then, $\lim _{\varepsilon \rightarrow 0} \lim _{t \rightarrow \infty} \mathbb{E}\left|\exp \left\{\bar{\xi}_{\varepsilon}(t)\right\}-\exp \{\bar{\xi}(t)\}\right|=0$. This yields

$$
\lim _{\varepsilon \rightarrow 0} \lim _{t \rightarrow \infty}\left|\bar{\xi}_{\varepsilon}(t)-\bar{\xi}(t)\right|=0, \text { in probalility. }
$$

And (41), (42) and (43) enable us to infer (39). To prove $\xi(t) \rightarrow \bar{\xi}$ in distribution as $t \rightarrow \infty$, by using (38), it suffices to show $\lim _{t \rightarrow \infty} \mathbb{E}[f(\bar{\xi})(t)-f(\xi)(t)]=0$, whenever $f$ is bounded and continuous on $\mathbb{R}$. Let such an $f$ be given, and set $M=\sup _{x \in \mathbb{R}}|f(x)|<\infty$. It follows from (38) that $\{\bar{\xi}(t)\}_{t} \geq 0$ is relatively compact. Then by Prohorov theorem [67], $\{\bar{\xi}(t)\}_{t} \geq 0$ is tight. So for each $\varepsilon>0$, there exists a compact set $K \subset \mathbb{R}$ such that $\mathbb{P}\left[\{\bar{\xi}(t) \in K] \geq 1-\frac{\epsilon}{6 M}\right.$ for any $t \geq 0$. Choose $0<\delta<1$ so $|f(x)-f(y)|<\frac{\varepsilon}{3}$ whenever $x \in K$ and $|x-y|<\delta$. From (39) we choose a positive number $T$ such that $\mathbb{P}[|\bar{\xi}(t)-\xi(t)| \geq \delta] \leq \frac{\varepsilon}{6 M}$ for all $t \geq T$, we have

$$
\begin{aligned}
\left|\int_{\Omega}[f(\bar{\xi})(t)-f(\xi)(t)] d \mathbb{P}\right| \leq & \frac{\varepsilon}{3} \mathbb{P}[\bar{\xi}(t) \in K,|\bar{\xi}(t)-\xi(t)|<\delta]+2 M \mathbb{P}[\bar{\xi}(t) \notin K] \\
& +2 M \mathbb{P}[\bar{\xi}(t) \in K,|\bar{\xi}(t)-\xi(t)| \geq \delta] \\
\leq & \varepsilon, \text { for all } t \geq T
\end{aligned}
$$


The proof is complete.

From Lemmas 4.9 and 4.10, the disease-free stationary distribution of stochastic model (6) can be shown in the following theorem.

Theorem 4.11. If $\mathcal{R}_{0}^{s}<1$, then $\lim _{t \rightarrow \infty} I(t)=0$ and $\lim _{t \rightarrow \infty} R(t)=0$ a.s., and the distribution of the process $X(t)$ converges to a disease-free stationary distribution which has the density defined as (30). Moreover, if $\frac{2 b d}{b+d}<\sigma_{1}^{2}<2 d$, the mean and variance of the stationary distribution of the process $X(t)$ are defined as in (31).

\subsection{Stochastic endemic dynamics}

The other of the main concerns in epidemiology is how we can regulate the endemic dynamics so that the disease will be persisted in a long term. From the above subsection, we know that Theorem 4.6 gives a condition $\mathcal{R}_{0}^{s}<1$ when solutions of model (6) are converging to the disease-free dynamics a.s.. Consider the condition $\mathcal{R}_{0}>1$ which plays the role of the endemic dynamics in the deterministic model (4), we can ask if there is a sharp condition $\mathcal{R}_{0}^{s}>1$ when solutions of model (6) are converging to the endemic dynamics a.s.. In addition, from Theorem 4.3, we know that the Markov process $X(t)$ of (6) with initial value $X_{0} \in \mathbb{R}_{+}^{3}$ is $V$-geometrically ergodic, hence there is a unique stationary distribution. And in this subsection, we will show that this unique stationary distribution of $X(t)$ of $(6)$ is endemic stationary distribution.

Theorem 4.12. Assume $\mathcal{R}_{0}^{s}>1$, if the following conditions are satisfied:

(i) $\max \left\{\frac{3 \mu-\delta}{2}, \sigma^{2}\right\}<d<b-\delta-\frac{1}{2} \sigma^{2}$;

(ii) $\Psi<\min \left\{\frac{d^{2} S^{* 2}}{d-\sigma_{1}^{2}}, \frac{4 d^{2} I^{* 2}}{2 d-\sigma_{2}^{2}}, \frac{4 d^{2}(2 d+\delta-\mu)^{2} R^{* 2}}{\mu\left(2 d(2 d+\delta-\mu)-\sigma_{3}^{2}(2 d+\delta+\mu)\right)}\right\}$, where $S^{*}, I^{*}, R^{*}$ are defined as in (13) and

$$
\Psi=\frac{d \sigma_{1}^{2} S^{* 2}}{d-\sigma_{1}^{2}}+\frac{2 d \sigma_{2}^{2} I^{* 2}}{2 d-\sigma_{2}^{2}}+\frac{2 d \sigma_{3}^{2}(2 d+\delta+\mu)(2 d+\delta-\mu) R^{* 2}}{\mu\left(2 d(2 d+\delta-\mu)-\sigma_{3}^{2}(2 d+\delta+\mu)\right)}+\frac{\epsilon}{2} I^{*} \sigma_{2}^{2},
$$


in which $\epsilon$ is sufficiently small positive, then there exists a unique stationary distribution $\pi(\cdot)$ for the stochastic model (6) with initial value $X_{0} \in \mathbb{R}_{+}^{3}$ and it has the ergodic property:

$$
\mathbb{P}\left\{\lim _{T \rightarrow \infty} \frac{1}{T} \int_{0}^{T} f\left(X_{i}(t)\right) d t=\int_{\Omega} z_{i} \pi\left(d z_{1}, d z_{2}, d z_{3}\right)\right\}=1, i=1,2,3 .
$$

Proof. Since $L$ defined in (8) is uniformly elliptical in $\mathbb{R}_{+}^{3}$, (P3) holds (see Chapter 3, p. 103 of Ref.[11] and Rayleigh's principle in [68], p.342). To verify (P4), it is enough to show that there exist some neighborhood $U$ and a non-negative $C^{2}$-function $V$ such that for any $X \in \mathbb{R}_{+}^{3} \backslash U, L V$ is negative definite function (for more details see p. 1163 in [69]).

Since $\mathcal{R}_{0}^{S}>1$, hence $\mathcal{R}_{0}=\mathcal{R}_{0}^{S}+\frac{\sigma_{2}^{2}}{2(d+\mu+\delta)}>1$, there is an endemic equilibrium $E^{*}=\left(S^{*}, I^{*}, R^{*}\right)$ of model (4). Then we have

$$
b=d S^{*}+\frac{k S^{* h} I^{*}}{S^{* h}+\alpha I^{* h}}-\gamma R^{*}, \frac{k S^{* h} I^{*}}{S^{* h}+\alpha I^{* h}}=(d+\mu+\delta) I^{*}, \quad \mu I^{*}=(d+\gamma) R^{*} .
$$

Define

$$
V(X)=V_{1}(X)+\epsilon V_{2}(x)+\frac{2 d+\delta}{\mu} V_{3}(X)
$$

where

$$
V_{1}(X)=\left(S-S^{*}+I-I^{*}+R-R^{*}\right)^{2}, V_{2}(X)=I-I^{*}-I^{*} \ln \frac{I}{I^{*}}, V_{3}(X)=\left(R-R^{*}\right)^{2} .
$$


Then $V$ is positive definited and $\lim _{|X| \rightarrow \infty} V(X)=\infty$. By virtue of (44), we can get

$$
\begin{aligned}
L V_{1}= & 2\left(S-S^{*}+I-I^{*}+R-R^{*}\right)\left(-d\left(S-S^{*}\right)-(d+\delta)\left(I-I^{*}\right)-d\left(R-R^{*}\right)\right) \\
& +\sigma_{1}^{2} S^{2}+\sigma_{2}^{2} I^{2}+\sigma_{3}^{2} R^{2} \\
= & -2 d\left(S-S^{*}\right)^{2}-2(d+\gamma)\left(I-I^{*}\right)^{2}-2 d\left(R-R^{*}\right)^{2}-2(2 d+\delta)\left(S-S^{*}\right)\left(I-I^{*}\right) \\
& -4 d\left(S-S^{*}\right)\left(R-R^{*}\right)-2(2 d+\delta)\left(I-I^{*}\right)\left(R-R^{*}\right)+\sigma_{1}^{2} S^{2}+\sigma_{2}^{2} I^{2}+\sigma_{3}^{2} R^{2}, \\
L V_{2}= & k\left(I-I^{*}\right)\left(\frac{S^{h}}{S^{h}+\alpha I^{h}}-\frac{S^{* h}}{S^{* h}+\alpha I^{* h}}\right)+\frac{1}{2} I^{*} \sigma_{2}^{2} \\
= & \frac{k S^{h}\left(I-I^{*}\right)\left[S^{* h}-S^{h}+\alpha\left(I^{* h}-I^{h}\right)\right]}{\left(S^{h}+\alpha I^{h}\right)\left(S^{* h}+\alpha I^{* h}\right)}+\frac{k\left(S^{h}-S^{* h}\right)\left(I-I^{*}\right)}{S^{* h}+\alpha I^{* h}}+\frac{1}{2} I^{*} \sigma_{2}^{2} \\
\leq & -\frac{k S^{h} A\left(S-S^{*}\right)\left(I-I^{*}\right)}{\left(S^{h}+\alpha I^{h}\right)\left(S^{* h}+\alpha I^{* h}\right)}+\frac{k A\left(S-S^{*}\right)\left(I-I^{*}\right)}{S^{* h}+\alpha I^{* h}}+\frac{1}{2} I^{*} \sigma_{2}^{2} \\
\leq & \frac{k C}{S^{* h}+\alpha I^{* h}}\left(S-S^{*}\right)\left(I-I^{*}\right) \operatorname{sgn}\left(S-S^{*}\right)\left(I-I^{*}\right)+\frac{1}{2} I^{*} \sigma_{2}^{2} \\
L V_{3}= & 2\left(R-R^{*}\right)\left[\mu\left(I-I^{*}\right)-(d+\gamma)\left(R-R^{*}\right)\right]+\sigma_{3}^{2} R^{2} \\
\leq & 2 \mu\left(R-R^{*}\right)\left(I-I^{*}\right)-2 d\left(R-R^{*}\right)^{2}+\sigma_{3}^{2} R^{2},
\end{aligned}
$$

This implies that

$$
\begin{aligned}
L V= & L V_{1}+\epsilon L V_{2}+\frac{2 d+\delta}{\mu} L V_{3} \\
\leq & -2 d\left(S-S^{*}\right)^{2}-2 d\left(I-I^{*}\right)^{2}-\frac{2 d(2 d+\delta+\mu)}{\mu}\left(R-R^{*}\right)^{2} \\
& +\sigma_{1}^{2} S^{2}+\sigma_{2}^{2} I^{2}+\frac{\sigma_{3}^{2}(2 d+\delta+\mu)}{\mu} R^{2}+\frac{\epsilon}{2} \sigma_{2}^{2} I^{*}+4 d\left|S-S^{*}\right|\left|R-R^{*}\right| \\
\leq & -d\left(S-S^{*}\right)^{2}-2 d\left(I-I^{*}\right)^{2}-\frac{2 d(2 d+\delta-\mu)}{\mu}\left(R-R^{*}\right)^{2} \\
& +\sigma_{1}^{2} S^{2}+\sigma_{2}^{2} I^{2}+\frac{\sigma_{3}^{2}(2 d+\delta+\mu)}{\mu} R^{2}+\frac{\epsilon}{2} \sigma_{2}^{2} I^{*} \\
= & -\left(d-\sigma_{1}^{2}\right)\left(S-\frac{d}{d-\sigma_{1}^{2}} S^{*}\right)^{2}-\left(2 d-\sigma_{2}^{2}\right)\left(I-\frac{2 d}{2 d-\sigma_{2}^{2}} I^{*}\right)^{2} \\
& -\left(\frac{2 d(2 d+\delta-\mu)}{\mu}-\frac{\sigma_{3}^{2}(2 d+\delta+\mu)}{\mu}\right)\left(R-\frac{2 d(2 d+\delta-\mu)}{2 d(2 d+\delta-\mu)-\sigma_{3}^{2}(2 d+\delta+\mu)} R^{*}\right)^{2}+\Psi \\
:= & -m_{1}\left(S-\theta_{1} S^{*}\right)^{2}-m_{2}\left(I-\theta_{2} I^{*}\right)^{2}-m_{3}\left(R-\theta_{3} R^{*}\right)^{2}+\Psi .
\end{aligned}
$$


Since $d>\frac{3 \mu-\delta}{2}, m_{2}>0$. Now if $\Psi$ satisfies the following conditions,

$$
0<\Psi<\min \left\{m_{1} \theta_{1}^{2} S^{* 2}, m_{2} \theta_{2}^{2} I^{* 2}, m_{3} \theta_{3}^{2} R^{* 2}\right\}
$$

then the ellipsoid

$$
m_{1}\left(S-\theta_{1} S^{*}\right)^{2}+m_{2}\left(I-\theta_{2} I^{*}\right)^{2}+m_{3}\left(R-\theta_{3} R^{*}\right)^{2}=\Psi
$$

lies entirely in $\mathbb{R}_{+}^{3}$. One can then take $U$ as any neighborhood of the ellipsoid such that $\bar{U} \subset \mathbb{R}_{+}^{3}$, where $\bar{U}$ is the closure of $U$. Thus, we have $L V(X)<0$ for $X \in \mathbb{R}_{+}^{3} \backslash U$ which implies condition (P4) in Lemma 2.8 is satisfied. As a consequence, the stochastic model (6) has a stationary distribution $\pi(\cdot)$ and it is ergodic.

According to the ergodic property, for $C>0$, we get

$$
\lim _{T \rightarrow \infty} \frac{1}{T} \int_{0}^{T}\left[X_{i}(t) \wedge C\right] d t=\int_{\mathbb{R}_{+}^{3}}\left[Z_{i} \wedge C\right] \pi\left(d Z_{1}, d Z_{2}, d Z_{3}\right), \text { a.s.. }
$$

In view of the dominated convergence theorem and $\mathbb{E}\left[X_{i}(t)\right]<C_{1}(i=1,2,3)$ which can obtained in Theorem 4.4, one can derive that

$$
\mathbb{E}\left[\lim _{T \rightarrow \infty} \frac{1}{T} \int_{0}^{T}\left[X_{i}(t) \wedge C\right] d t\right]=\lim _{T \rightarrow \infty} \frac{1}{T} \int_{0}^{T} \mathbb{E}\left[X_{i}(t) \wedge C\right] d t \leq C_{1} .
$$

It follows that

$$
\int_{\mathbb{R}_{+}^{3}}\left[Z_{i} \wedge C\right] \pi\left(d Z_{1}, d Z_{2}, d Z_{3}\right) \leq C_{1} .
$$

Letting $C \rightarrow \infty$ results in $\int_{\mathbb{R}_{+}^{3}} Z_{i} \pi\left(d Z_{1}, d Z_{2}, d Z_{3}\right) \leq C_{1}$. Thus the function $f(X)=X$ is integrable with respect to the measure $\pi(\cdot)$. Then the desired assertion follows from Lemma 2.8 immediately.

Remark 4.13. Theorem 4.12 gives the possibility that an asymptotically stationary distribution exists for the solution of model (6) which in turn implies the stability in a stochastic sense. Furthermore, Theorem 4.12 suggests that if the condition of the theorem is satisfied, 
then the stochastic model (6) oscillates around the endemic equilibrium $E^{*}$ of the deterministic model (4), and model (6) has the ergodic property where the positive solution converges to the unique stationary distribution. This reveals the persistence of the disease a.s. under certain conditions.

\section{Numerical simulations and dynamics comparison}

In this section, we give some numerical simulations to show the effect of noise on the dynamics of the SIRS models by using the Milstein method mentioned in Higham [70]. In this way, the SDE model (6) can be rewritten as the following discretization equations:

$$
\left\{\begin{array}{l}
S_{k+1}=S_{k}+\left(b-d S-\frac{k S^{h} I}{S^{h}+\alpha I^{h}}+\gamma R\right) \Delta t+\sigma_{1} S_{k} \sqrt{\Delta t} \xi_{k}+\frac{\sigma_{1}^{2}}{2} S_{k}\left(\xi_{k}^{2}-1\right) \Delta t \\
I_{k+1}=I_{k}+\left(\frac{k S^{h} I}{S^{h}+\alpha I^{h}}-(d+\mu+\delta) I\right) \Delta t+\sigma_{2} I_{k} \sqrt{\Delta t} \eta_{k}+\frac{\sigma_{2}^{2}}{2} I_{k}\left(\eta_{k}^{2}-1\right) \Delta t \\
R_{k+1}=R_{k}+(\mu I-(d+\gamma) R) \Delta t+\sigma_{3} R_{k} \sqrt{\Delta t} \zeta_{k}+\frac{\sigma_{3}^{2}}{2} R_{k}\left(\zeta_{k}^{2}-1\right) \Delta t
\end{array}\right.
$$

where $\xi_{k}, \eta_{k}$ and $\zeta_{k}, k=1,2, \cdots, n$, are the Gaussian random variables $N(0,1)$. The main goal of this section is to further investigate the answers to the following three questions as we proposed in the introduction:

1. Compare different dynamic outcomes of the deterministic model (4) v.s. its stochastic version (6).

2. Two different stationary distributions of the solutions of stochastic model (6) under different conditions.

\subsection{Stochasticity suppresses the disease outbreak}

In this subsection, we give an example to show different dynamic outcomes of the deterministic model (4) v.s. its stochastic version (6) with the same set of parameter values. 
Example 5.1. For the deterministic model (4) and its stochastic model (6), the parameters are taken as follows

$$
b=1, d=0.1, k=0.2, \alpha=0.5, \gamma=0.01, \mu=0.05, \delta=0.01, h=2 .
$$

1. For the deterministic model $(4), \mathcal{R}_{0}=\frac{k}{d+\mu+\delta}=1.25>1$, thus it admits a unique endemic equilibrium $E^{*}=(4.7637,3.3684,1.5311)$ which is globally stable for any initial values $(S(0), I(0), R(0)) \in \Gamma$ according to Theorem 3.3 (see, Fig. 1(a)).

Fig.1

2. For the corresponding stochastic model (6), we choose $\sigma_{1}=0.2, \sigma_{2}=0.3, \sigma_{3}=0.1$, then we have

$$
\mathcal{R}_{0}^{s}=\frac{k}{d+\mu+\delta}-\frac{\sigma_{2}^{2}}{2(d+\mu+\delta)}=0.9688<1 .
$$

Then according to Theorem 4.6, we can conclude that for any initial value $(S(0), I(0), R(0)) \in$ $\mathbb{R}_{+}^{3}, I(t)$ obeys

$$
\limsup _{t \rightarrow \infty} \frac{\log I(t)}{t} \leq-0.005 \text { a.s.. }
$$

That is, $I(t)$ will tend to zero exponentially with probability one (see Fig. 1(b)). To see the disease dynamics of (6) when $\mathcal{R}_{0}^{s}>1$, we decrease $\sigma_{2}$ to 0.2 , and keep other parameters unchanged. Then we have

$$
\mathcal{R}_{0}^{s}=\frac{k}{d+\mu+\delta}-\frac{\sigma_{2}^{2}}{2(d+\mu+\delta)}=1.125>1 .
$$

Therefore, the condition of Theorem 4.6 is not satisfied. In this case, our simulations suggest that $I(t)$ is stochastically persistent (see Fig. 1(c)).

Brief summary: Theorem 3.3 and 4.6 as well as numerical simulations (see examples illustrated in Fig.1) indicate that environmental fluctuations can suppress the disease outbreak. In the case of $\mathcal{R}_{0}>1$, the deterministic model (4) admits a globally stable endemic equilibrium $E^{*}$, while introducing large noise intensity $\sigma_{2}$ in $I$ of the stochastic model (6) can 
eradicate the disease a.s.. Theorem 4.4 indicates that the norm of $(S(t), I(t), R(t))$ of model (6) is bounded away from extinction almost surely whenever the condition $2 d+\delta<b$ holds. The parameters of the two cases of model (6) (i.e., Figures 1(b) and (c)) satisfy the conditions, and they both are stochastically permanent. Even though Theorem 4.4 doesn't give us any information whether $S(t) I(t) R(t)>0$ or not, simulations (e.g., Figures 1 ) suggest that the persistence of infectious $I$ can be governed by the value of $\mathcal{R}_{0}^{S}$ : if $\mathcal{R}_{0}^{S}<1, I$ is extinction a.s. (e.g., Fig.1(b)), while if $\mathcal{R}_{0}^{S}>1, I$ is stochastically persistent (e.g., Fig.1(c)). We have invested more on the case of $\mathcal{R}_{0}^{S}>1$ in the next subsection.

\subsection{Stationary distribution}

In this subsection, we further investigate two stationary distributions of the SDE model (6) governed by the value $\mathcal{R}_{0}^{s}$ : the SDE model (6) has disease-free stationary distribution when $\mathcal{R}_{0}^{s}<1$; while it has an endemic stationary distribution when $\mathcal{R}_{0}^{s}>1$.

1. The unique disease-free stationary distribution occurs when $\mathcal{R}_{0}^{S}<1$ according to Theorem 4.6 where both $I(t)$ and $R(t)$ go extinct exponentially a.s.. This leads to the situation that $S(t)$ has a unique stationary distribution according to Theorem 4.11. To illustrate the disease-free stationary distribution, we provide numerical results in Fig.2 whose parameters are the same as those in Fig.1(b).

Example 5.2. We chose the parameters taken as (47) and choose $\sigma_{1}=0.44, \sigma_{2}=$ $0.9, \sigma_{3}=0.1$. In this case, $\mathcal{R}_{0}^{s}=0.9688<1$. And we present the frequency histograms based on 10000 stochastic simulations for $S(t), I(t)$ and $R(t)$ at time $t=200$ by using the Statistical Software R in Fig.2, and the numerical method for them can be found in [18]. One can see that $I(t)$ and $R(t)$ go extinct exponentially a.s., and $S(t)$ has a unique stationary distribution.

Fig.2 
2. The endemic stationary distribution occurs when $\mathcal{R}_{0}^{S}>1$ suggested by Theorem 4.12 .

Example 5.3. To investigate the endemic stationary distribution, we present the frequency histograms based on 10000 stochastic simulations for $S(t), I(t)$ and $R(t)$ at time $t=200$ by using the Statistical Software R, where the parameters are taken as (47) and $\sigma_{1}=0.01, \sigma_{2}=0.02, \sigma_{3}=0.01$ (see Fig.3). In this case, we have

$$
\frac{d^{2} S^{* 2}}{d-\sigma_{1}^{2}}=2.2715, \frac{4 d^{2} I^{* 2}}{2 d-\sigma_{2}^{2}}=2.2738, \frac{4 d^{2}(2 d+\delta-\mu)^{2} R^{* 2}}{\mu\left(2 d(2 d+\delta-\mu)-\sigma_{3}^{2}(2 d+\delta+\mu)\right)}=9.3846
$$

and

$$
\frac{d \sigma_{1}^{2} S^{* 2}}{d-\sigma_{1}^{2}}+\frac{2 d \sigma_{2}^{2} I^{* 2}}{2 d-\sigma_{2}^{2}}+\frac{2 d \sigma_{3}^{2}(2 d+\delta+\mu)(2 d+\delta-\mu) R^{* 2}}{\mu\left(2 d(2 d+\delta-\mu)-\sigma_{3}^{2}(2 d+\delta+\mu)\right)}=0.008 .
$$

Hence, for sufficiently small $\epsilon$ such that $\Psi<\min \{2.2715,2.2738,9.3846\}$. Thus according to Theorem 4.12, we can conclude that model (6) has a unique endemic stationary distribution.

\section{Fig.3}

If $\mathcal{R}_{0}^{S}>1$, the SDE model (6) has a unique endemic stationary distribution as the solution of stochastic model (6) around endemic $E^{*}$ of model (4). Fig.3 as examples illustrates the existence and uniqueness of the stationary distribution of $S(t), I(t)$ and $R(t)$. To further explore the effect of the intensity of noises, we choose the same main parameters as those in Fig. 3 but with different intensity of noise. In this case, we still have $\mathcal{R}_{0}=1.25$, thus the deterministic model (4) admits a unique endemic equilibrium $E^{*}=(4.7637,3.3684,1.5311)$ which is globally stable according to Theorem 3.3. Let $\sigma_{1}=0.01, \sigma_{2}=0.02, \sigma_{3}=0.01$, then we have $\mathcal{R}_{0}^{s}=1.2488>1$. If we keep all the parameters in (47) unchanged but increase $\left(\sigma_{1}, \sigma_{2}, \sigma_{3}\right)$ to $(0.1,0.2,0.1)$, then we have $\mathcal{R}_{0}^{s}=1.125>1$. Our Theorem 4.12 and Remark 4.13 indicate that the solutions of the stochastic model (6) are oscillating around the endemic equilibrium $E^{*}=(4.7637,3.3684,1.5311)$ of model $(4)$ for both cases $\left(\sigma_{1}, \sigma_{2}, \sigma_{3}\right)=$ $(0.01,0.02,0.01)$ (see Figure $4(\mathrm{a}))$ and $\left(\sigma_{1}, \sigma_{2}, \sigma_{3}\right)=(0.1,0.2,0.1)$ (see Figure $\left.4(\mathrm{~b})\right)$. 


\section{Fig.4}

\section{Discussions}

Fluctuations in the natural environment introduce variability into the biological systems that exist within them [16]. In many instances, environmental variations have a critical influence on the development of an epidemic $[11,12,13,14,15,16,17,20]$. For example, many species of fungi have threshold temperatures and humidity levels for germination to occur [16]. Variability of temperature and rainfall may induce pronounced fluctuations in the dynamics of pathogenic fungi [19]. For human disease, the nature of epidemic growth and spread is inherently random due to the unpredictability of person-to-person contacts [8] and population is subject to a continuous spectrum of disturbances $[9,10]$. Hence the variability and randomness of the environment is fed through to the state of the epidemic [16]. In this article, we focus on a stochastic differential equation SIRS model with ratio-dependent incidence rate.

Among the various ways of constructing a stochastic model systems for a given deterministic system, we propose a stochastic version of the SIRS epidemic model with nonlinear incident rate. In our model (6), we suppose that stochastic environmental factor acts simultaneously on each individual in the population, and assume that the stochastic perturbation is a white noise type that is influenced on the the natural death rate $d$. This is a well-established way of introducing stochastic environmental noise into biologically realistic population dynamic models that have been used in $[25,26,35,36,40,42,46]$.

The value of our study lies in two aspects: Mathematically, we show that the global dynamics of the deterministic model (4) can be governed by its reproduction number $R_{0}$

while the dynamics of its stochastic version (6) seem to be governed by $\mathcal{R}_{0}^{S}=\mathcal{R}_{0}\left(1-\frac{\sigma_{2}^{2}}{2 k}\right)$. In addition, we have provided the analytic results on the existence of global positive solution, stochastic boundedness, permanence/extinction, asymptotic stability and ergodic property 
of the solution of the SDE model (6). Epidemiologically, we partially provide answers to the two questions proposed in the introduction: What is the disease dynamics of stochastic SIRS model with ratio-dependent incidence rate? How may different types of environmental fluctuations produce different dynamical outcomes? We summarize our main findings as follows:

1. Noise can suppress the disease outbreak: Theorem 4.6 indicates that the extinction of disease in the stochastic model (6) occurs if the basic reproduction number $\mathcal{R}_{0}^{S}=$ $\mathcal{R}_{0}-\frac{\sigma_{2}^{2}}{2(d+\mu+\delta)}<1$. Theorem 3.3 shows that the deterministic model (4) admits a unique endemic equilibrium $E^{*}$ which is globally asymptotically stable if its basic reproduction number $\mathcal{R}_{0}>1$. Notice that $\mathcal{R}_{0}^{S}<\mathcal{R}_{0}$, and it is possible that $\mathcal{R}_{0}^{S}<1<\mathcal{R}_{0}$. This is the case when the deterministic model (4) has an endemic (see Fig. 1(a)) while the stochastic model (6) has disease extinction with probability one (see Fig. 1(b)). This implies that large environment fluctuations in I-class can suppress the outbreak of disease, which partially answer the first question proposed in the introduction.

2. The stationary distributions governed by $\mathcal{R}_{0}^{S}$ : As suggested by Theorems $4.11,4.12$ and simulations (Figures 2 and 3), model (6) has two kinds of stationary distributions depending on the value of $\mathcal{R}_{0}^{S}$ : If $\mathcal{R}_{0}^{S}<1$, it has disease-free stationary distribution (see Fig.2) which means that the disease will die out with probability one; while if $\mathcal{R}_{0}^{S}>1$ it has endemic stationary distribution (c.f., Fig.3). The latter leads to the stochastically persistence of the disease. The numerical results in Fig.3 suggest the existence and uniqueness of the endemic stationary distribution of $S(t), I(t)$ and $R(t)$. These partially answer the second question proposed in the introduction.

Furthermore, from Theorems 4.11,4.12 and numerical results (e.g., Figures 1 and 4), we can conclude that, when the intensity of noise is small, the stochastic model preserves the property of the global stability. In this case, we can ignore noise and use the deterministic model to approximate the population dynamics. However, the large intensity of noise 
can force the solutions of model (6) to oscillate strongly around the disease-free (c.f., Theo-

rem 4.11) or endemic points (c.f., Theorem 4.12 and Fig.4), or the extinct (c.f., Theorem 4.6 and Fig.1(c)). In these cases, we cannot ignore the effect of noise and, therefore, we cannot use deterministic model but stochastic model to describe the disease dynamics.

In addition, we notice that $h$ is independent on the existence of the endemic point $E^{*}$ of model (4) but dependent on the value of $E^{*}$ (see the equilibrium expression (13)). We would like to point out that all theorems related to the deterministic model (4) and the stochastic model (6) (except Theorem 4.12), are not depend on the parameter $h$, for Theorem 4.12 is related to $E^{*}=\left(S^{*}, I^{*}, R^{*}\right)$. While others are independent on $h$ during the proving process, we amplify the values too large to contain $h$ by using the method of amplification.

\section{Acknowledgements}

This research was supported by the National Science Foundation of China (11601179\& 61373005 \& 61672013 ), the Natural Science Foundation of the Jiangsu Higher Education Institutions of China (16KJB110003). The research of Y. Kang was partially supported by NSF-DMS (1313312) and the research scholarship from School of Letters and Sciences.

\section{References}

[1] World Health Organization. The top 10 causes of death. http:www.who.int/mediacentre /factsheets/fs310/en/index.html, 2011.

[2] W.O. Kermack and A.G. McKendrick. Contributions to the mathematical theory of epidemics-I. Proceedings of the Royal Society of London A, 115(772):701-721, 1927.

[3] Z. Ma, Y. Zhou, and J. Wu. Modeling and dynamics of infectious diseases. Higher Education Press, 2009.

[4] M. Keeling and P. Rohani. Modeling infectious diseases in humans and animals. Princeton University Press, 2008. 
[5] M.D.L. Sen, R.P. Agarwal, A. Ibeas, and S. Alonso-Quesada. On a generalized timevarying SEIR epidemic model with mixed point and distributed time-varying delays and combined regular and impulsive vaccination controls. Advances in Difference Equations, 2010: 281612, 2010.

[6] M.D.L. Sen, S. Alonso-Quesadaa, and A. Ibeas. On the stability of an SEIR epidemic model with distributed time-delay and a general class of feedback vaccination rules. Applied Mathematics and Computation, 270: 953-976, 2015.

[7] H. C. Tuckwell and R. J. Williams. Some properties of a simple stochastic epidemic model of SIR type. Mathematical Biosciences, 208(1):76-97, 2007.

[8] S. Spencer. Stochastic epidemic models for emerging diseases. PhD thesis, University of Nottingham, 2008.

[9] J.R. Beddington and R.M. May. Harvesting natural populations in a randomly fluctuating environment. Science, 197(4302):463-465, 1977.

[10] L.J.S. Allen. An introduction to stochastic epidemic models. In Mathematical Epidemiology, pages 81-130. Springer, 2008.

[11] C.G. Thomas. Introduction to stochastic differential equations. Dekker, New York, 1988.

[12] X. Mao. Stochastic differential equations and their applications (Second Edition). Horwood, Chichester, UK 2007.

[13] R. Durrett. Stochastic spatial models. SIAM Review, 41(4):677-718, 1999.

[14] R.Z. Khasminskii and F.C. Klebaner. Long term behavior of solutions of the LotkaVolterra system under small random perturbations. Annals of Applied Probability, 11(3):952-963, 2001. 
[15] X. Mao, G. Marion, and E. Renshaw. Environmental brownian noise suppresses explosions in population dynamics. Stochastic Processes and Their Applications, 97(1):95$110,2002$.

[16] J.E. Truscott and C.A. Gilligan. Response of a deterministic epidemiological system to a stochastically varying environment. Proceedings of the National Academy of Sciences, 100(15):9067-9072, 2003.

[17] B. Arifah, and X. Mao. Stochastic delay Lotka-Volterra model. Journal of Mathematical Analysis and Applications, 292(2): 364-380,2004

[18] Y. Cai, Y. Kang, M. Banerjee, and W. Wang, A stochastic SIRS epidemic model with infectious force under intervention strategies, Journal of Differential Equations, 259:7463$7502,2015$.

[19] M. Liu, Global asymptotic stability of stochastic Lotka-Volterra systems with infinite delays, IMA Journal of Applied Mathematics 80(2015)1431-1453.

[20] D. Li, J. Cui, M. Liu, and S. Liu. The evolutionary dynamics of stochastic epidemic model with nonlinear incidence rate. Bulletin of Mathematical Biology, 77(9):1705-1743, 2015 .

[21] Y. Cai, Y. Kang, M. Banerjee, and W. Wang. A stochastic epidemic model incorporating media coverage. Communications in Mathematical Sciences, 14(4):893-910, 2016.

[22] D. Jiang, N. Shi, and X. Li. Global stability and stochastic permanence of a nonautonomous Logistic equation with random perturbation. Journal of Mathematical Analysis and Applications, 340(1):588-597, 2008.

[23] X. Li, and X. Mao. Population dynamical behavior of non-autonomous lotka-volterra competitive system with random perturbation. Discrete and Continuous Dynamical Systems, 24(2):523-593, 2009. 
[24] M. Liu and M. Fan. Permanence of Stochastic Lotka-Volterra Systems. Journal of Nonlinear Science, doi:10.1007/s00332-016-9337-2, 2016.

[25] A. Lahrouz and L. Omari. Extinction and stationary distribution of a stochastic SIRS epidemic model with non-linear incidence. Statistics \& Probability Letters, 83:960-968, 2013.

[26] Q. Yang and X. Mao. Stochastic dynamics of SIRS epidemic models with random perturbation. Mathematical Biosciences and Engineering, 11(4):1003-1025, 2014.

[27] N. Du, N. Nhu. Permanence and extinction of certain stochastic SIR models perturbed by a complex type of noises, Applied Mathematics Letters, 64:223-230, 2017.

[28] C. Xu. Global threshold dynamics of a stochastic differential equation SIS model, Journal of Mathematical Analysis and Applications, 447:736-757, 2017.

[29] Q. Liu, D. Jiang. The threshold of a stochastic delayed SIR epidemic model with vaccination, Physica A, 461:140-147, 2016.

[30] D. Zhao. Study on the threshold of a stochastic SIR epidemic model and its extensions, Communications in Nonlinear Science and Numerical Simulation, 38:172-177, 2016.

[31] J. Li, M. Shan, M. Banerjee, W. Wang. Stochastic dynamics of feline immunodeficiency virus within cat populations, Journal of the Franklin Institute, 353:4191-4212, 2016.

[32] Y. Liu, M. Shan, X. Lian, W. Wang. Stochastic extinction and persistence of a parasiteChost epidemiological model, Physica A, 462:586-602, 2016.

[33] F. Xie, M. Shan, X. Lian, W. Wang. Periodic solution of a stochastic HBV infection model with logistic hepatocyte growth, Applied Mathematics and Computation, 293:630-641, 2017. 
[34] T. Britton and D. Lindenstrand. Epidemic modelling: Aspects where stochastic epidemic models: A survey. Mathematical Biosciences, 222(1):109-116, 2010.

[35] N. Dalal, D. Greenhalgh, and X. Mao. A stochastic model of AIDS and condom use. Journal of Mathematical Analysis and Applications, 325(1):36-53, 2007.

[36] N. Dalal, D. Greenhalgh, and X. Mao. A stochastic model for internal HIV dynamics. Journal of Mathematical Analysis and Applications, 341(2):1084-1101, 2008.

[37] T. Britton. Stochastic epidemic models: A survey. Mathematical Biosciences, 225(1):24$35,2010$.

[38] F. Ball and P. Neal. A general model for stochastic SIR epidemics with two levels of mixing. Mathematical Biosciences, 180:73-102, 2002.

[39] F. Ball, D. Sirl, and P. Trapman. Analysis of a stochastic SIR epidemic on a random network incorporating household structure. Mathematical Biosciences, 224(2):53-73, 2010.

[40] A. Gray, D. Greenhalgh, L. Hu, X. Mao, and J. Pan. A stochastic differential equation SIS epidemic model. SIAM Journal on Applied Mathematics, 71(3):876-902, 2011.

[41] A. Lahrouz, L. Omari, and D. Kiouach. Global analysis of a deterministic and stochastic nonlinear SIRS epidemic model. Nonlinear Analysis: Modelling and Control, 16(1):59$76,2011$.

[42] Z. Liu. Dynamics of positive solutions to SIR and SEIR epidemic models with saturated incidence rates. Nonlinear Analysis: Real World Applications, 14(3):1286-1299, 2013.

[43] Y. Cai, X. Wang, W. Wang, and M. Zhao. Stochastic dynamics of a SIRS epidemic model with ratio-dependent incidence rate. Abstract and Applied Analysis, 2013:172631, 2013. 
[44] O.A. van Herwaarden and J. Grasman. Stochastic epidemics: major outbreaks and the duration of the endemic period. Journal of Mathematical Biology, 33(6):581-601, 1995.

[45] L.J.S. Allen. An introduction to stochastic processes with applications to biology. Pearson Education New Jersey, 2003.

[46] Q. Yang, D. Jiang, N. Shi, and C. Ji. The ergodicity and extinction of stochastically perturbed SIR and SEIR epidemic models with saturated incidence. Journal of Mathematical Analysis and Applications, 388(1):248-271, 2012.

[47] R.M. Anderson and R. M. May. Population biology of infectious diseases: Part I. Nature, 280(5721):361, 1979.

[48] B.B. Mukhopadhyay and P.K. Tapaswi. An SIRS epidemic model of Japanese encephalitis. International Journal of Mathematics and Mathematical Sciences, 17(2):347-355, 1994.

[49] J. Tumwiine, J.Y.T. Mugisha, and L.S. Luboobi. A mathematical model for the dynamics of malaria in a human host and mosquito vector with temporary immunity. Applied Mathematics and Computation, 189(2):1953-1965, 2007.

[50] C. Bain. Applied mathematical ecology. Journal of Epidemiology and Community Health, 44(3):254, 1990.

[51] A. Korobeinikov and P.K. Maini. Non-linear incidence and stability of infectious disease models. Mathematical Medicine and Biology, 22(2):113-128, 2005.

[52] H.W. Hethcote. The mathematics of infectious diseases. SIAM Review, 42(4):599-653, 2000.

[53] B. Fred and C.-C. Carlos. Mathematical models in population biology and epidemiology (Second Edition). Springer, 2012. 
[54] V. Capasso and G. Serio. A generalization of the Kermack-Mckendrick deterministic epidemic model. Mathematical Biosciences, 42(1):43-61, 1978.

[55] W. Liu, S.A. Levin, and Y. Iwasa. Influence of nonlinear incidence rates upon the behavior of SIRS epidemiological models. Journal of Mathematical Biology, 23(2):187204, 1986.

[56] S. Yuan and B. Li. Global dynamics of an epidemic model with a ratio-dependent nonlinear incidence rate. Discrete Dynamics in Nature and Society, 2009, 2009.

[57] R.M. May. Stability and complexity in model ecosystems. Princeton University Press, 1973.

[58] L.R. Bellet. Ergodic properties of markov processes. In Open Quantum Systems II, pages 1-39. Springer, 2006.

[59] S.P. Meyn and R.L. Tweedie. Stability of markovian processes III: Foster-Lyapunov criteria for continuous-time processes. Advances in Applied Probability, pages 518-548, 1993.

[60] J.C. Mattingly, A.M. Stuart, and D.J. Higham. Ergodicity for SDES and approximations: locally Lipschitz vector fields and degenerate noise. Stochastic processes and their applications, 101(2):185-232, 2002.

[61] R. Khasminskii. Stochastic stability of differential equations (Second Edition), volume 66. Springer-Verlag Berlin Heidelberg, 2012.

[62] P. Driessche and J. Watmough. Reproduction numbers and sub-threshold endemic equilibria for compartmental models of disease transmission. Mathematical Biosciences, 180(1):29-48, 2002. 
[63] J.P. LaSalle. The stability of dynamical systems, Regional Conference Series in Applied Mathematics, Society for Industrial and Applied Mathematics, Philadelphia, 1976.

[64] A. M. Lyapunov. The general problem of the stability of motion. International Journal of Control, 55(3):531-773, 1992.

[65] A. Athreya, T. Kolba, J.C. Mattingly. Propagating Lyapunov functions to prove noiseinduced stabilization. Electronic Journal of Probability, 17(96): 1-38, 2012.

[66] A.V. Skorokhod. Asymptotic methods in the theory of stochastic differential equations, volume 78. American Mathematical Soc., 2009.

[67] I. Karatzas and S. Shreve Brownian motion and stochastic calculus, Springer-Verlag, New York Inc., 1988.

[68] G. Strang. Linear Algebra and its Applications (Fourth Edition). Thomson Learning, Inc., 2006.

[69] C. Zhu and G. Yin. Asymptotic properties of hybrid diffusion systems. SIAM Journal on Control and Optimization, 46(4):1155-1179, 2007.

[70] D.J. Higham. An algorithmic introduction to numerical simulation of stochastic differential equations. SIAM Review, 43(3):525-546, 2001. 


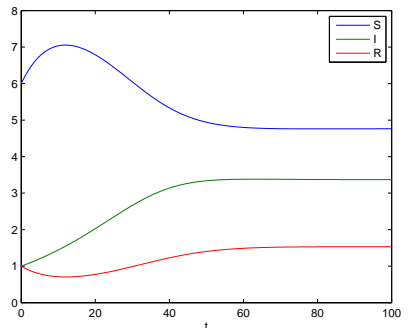

(a) $\sigma_{1}=0, \sigma_{2}=0, \sigma_{3}=0$

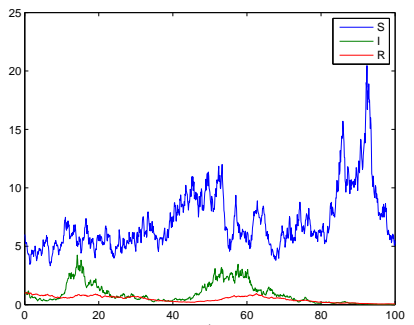

(b) $\sigma_{1}=0.2, \sigma_{2}=0.3, \sigma_{3}=0.1$

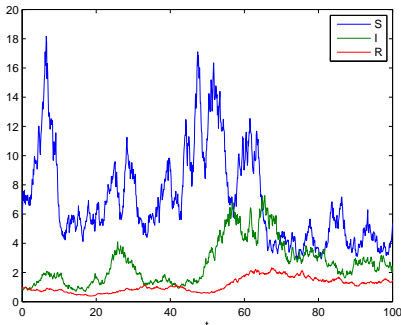

(c) $\sigma_{1}=0.2, \sigma_{2}=0.2, \sigma_{3}=0.1$

Figure 1: The path $S(t), I(t)$ and $R(t)$ for the stochastic model (6) with initial values $(S(0), I(0), R(0))=$ $(6,1,1)$. The parameters are taken as $(47)$.

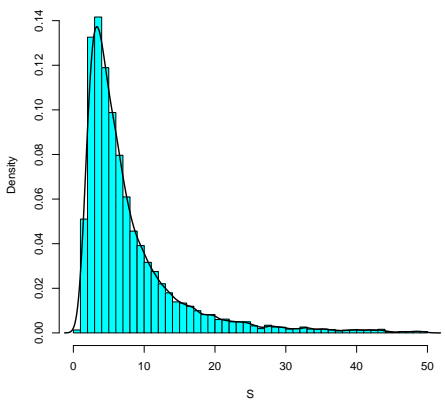

(a)

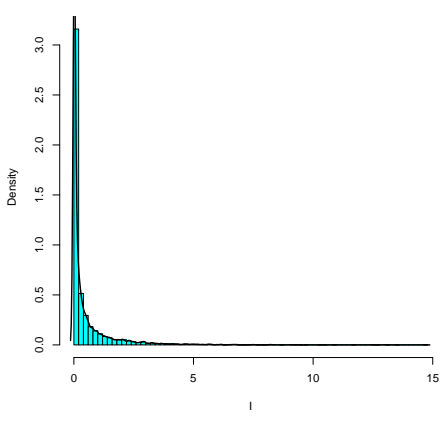

(b)

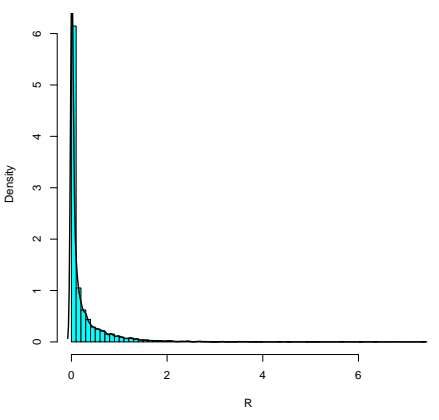

(c)

Figure 2: Frequency histograms based on 10000 stochastic simulations for each population at time $t=200$. The parameter values are the same as them in Fig.1(b). 


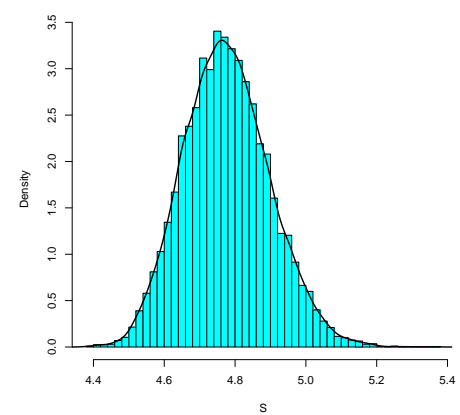

(a)

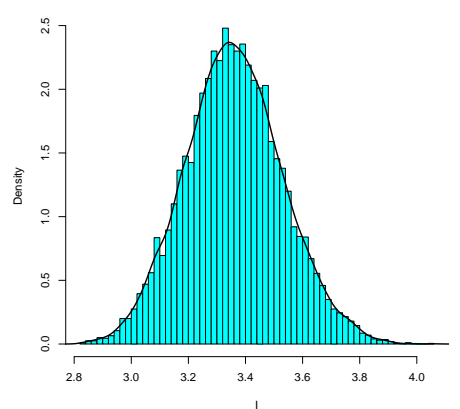

(b)

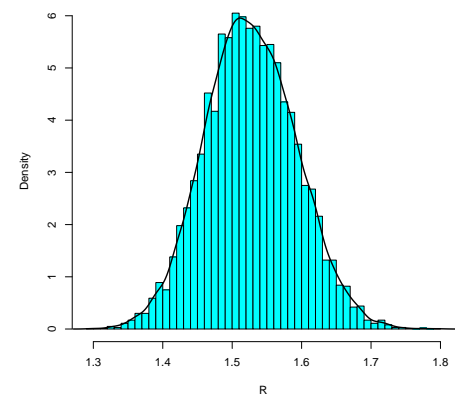

(c)

Figure 3: Frequency histograms based on 10000 stochastic simulations for each population at time $t=200$.

The parameter values in (a),(b) and (c) are in (47) and $\sigma_{1}=0.01, \sigma_{2}=0.02, \sigma_{3}=0.01$.

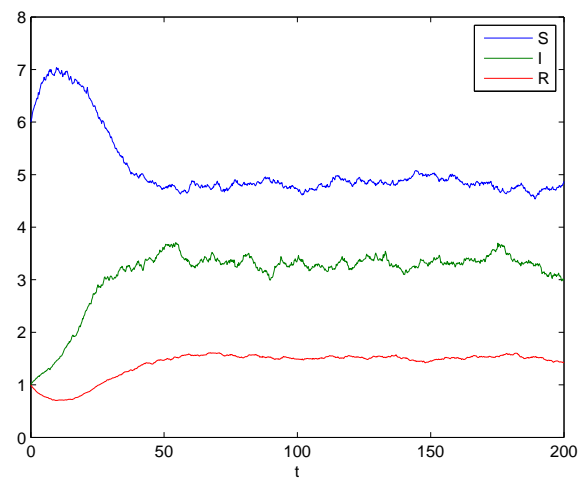

(a) $\sigma_{1}=0.01, \sigma_{2}=0.02, \sigma_{3}=0.01$

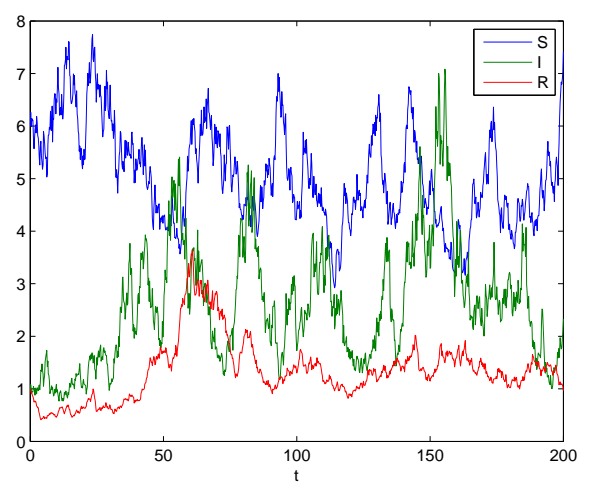

(b) $\sigma_{1}=0.1, \sigma_{2}=0.2, \sigma_{3}=0.1$

Figure 4: The asymptotic behavior of the solutions to the stochastic model (6) with parameters (47) around the endemic equilibrium $E^{*}=(4.7637,3.3684,1.5311)$ of model $(4)$ with initial values $S(0)=6, I(0)=$ $1, R(0)=1$. 